\title{
Baryon Acoustic Oscillations in the Sloan Digital Sky Survey Data Release 7 Galaxy Sample
}

\author{
Will J. Percival ${ }^{1 \star}$, Beth A. Reid ${ }^{2,3}$, Daniel J. Eisenstein ${ }^{4}$, Neta A. Bahcall ${ }^{3}$, Tamas \\ Budavari $^{5}$, Joshua A. Frieman ${ }^{6,7}$, Masataka Fukugita ${ }^{8}$, James E. Gunn ${ }^{3}$, Željko Ivezić ${ }^{9}$, \\ Gillian R. Knapp ${ }^{3}$, Richard G. Kron ${ }^{10}$, Jon Loveday ${ }^{11}$, Robert H. Lupton ${ }^{3}$, Timothy A. \\ McKay ${ }^{12}$, Avery Meiksin ${ }^{13}$, Robert C. Nichol ${ }^{1}$, Adrian C. Pope ${ }^{14}$, David J. Schlegel ${ }^{15}$, \\ Donald P. Schneider ${ }^{16}$, David N. Spergel ${ }^{3,17}$, Chris Stoughton ${ }^{18}$, Michael A. Strauss ${ }^{3}$, \\ Alexander S. Szalay ${ }^{5}$, Max Tegmark ${ }^{19}$, Michael S. Vogeley ${ }^{20}$, David H. Weinberg ${ }^{21}$, \\ Donald G. York ${ }^{10,22}$, Idit Zehavi ${ }^{23}$, \\ ${ }^{1}$ Institute of Cosmology and Gravitation, University of Portsmouth, Dennis Sciama building, Portsmouth, P01 3FX, UK \\ ${ }^{2}$ Institute of Space Sciences (CSIC-IEEC), UAB, Barcelona 08193, Spain and \\ Institute for Sciences of the Cosmos (ICC), University of Barcelona, Barcelona 08028, Spain \\ ${ }^{3}$ Department of Astrophysical Sciences, Princeton University, Princeton, NJ 08544, USA \\ ${ }^{4}$ Steward Observatory, University of Arizona, 933 N. Cherry Ave., Tucson, AZ 85121, USA \\ ${ }^{5}$ Department of Physics and Astronomy, The Johns Hopkins University, 3701 San Martin Drive, Baltimore, MD 21218, USA \\ ${ }^{6}$ Particle Astrophysics Center, Fermilab, P.O. Box 500, Batavia, IL 60510, USA \\ ${ }^{7}$ Kavli Institute for Cosmological Physics, Department of Astronomy \& Astrophysics, University of Chicago, Chicago, IL 60637, USA \\ ${ }^{8}$ Institute for Cosmic Ray Research, University of Tokyo, Kashiwa 277-8582, Japan \\ ${ }^{9}$ Department of Astronomy, University of Washington Box 351580, Seattle, WA 98195, USA \\ ${ }^{10}$ Department of Astronomy and Astrophysics, The University of Chicago, 5640 South Ellis Avenue, Chicago, IL 60637, USA \\ ${ }^{11}$ Astronomy Centre, University of Sussex, Falmer, Brighton, BN1 9QH, UK \\ ${ }^{12}$ Departments of Physics and Astronomy, University of Michigan, Ann Arbor, MI, 48109, USA \\ ${ }^{13}$ SUPA; Institute for Astronomy, University of Edinburgh, Royal Observatory, Blackford Hill, Edinburgh, EH9 3HJ, UK \\ ${ }^{14}$ Los Alamos National Laboratory, PO Box 1663, Los Alamos, NM 87545, USA \\ ${ }^{15}$ Lawrence Berkeley National Lab, 1 Cyclotron Road, MS 50R5032, Berkeley, CA94720, USA \\ ${ }^{16}$ Department of Astronomy and Astrophysics, The Pennsylvania State University, University Park, PA 16802, USA \\ ${ }^{17}$ Princeton Center for Theoretical Science, Princeton University, Jadwin Hall, Princeton, NJ 08542 USA \\ ${ }^{18}$ Fermilab, PO Box 500, Batavia, IL 60510, USA \\ ${ }^{19}$ Department of Physics, Massachusetts Institute of Technology, Cambridge, MA 02139, USA \\ ${ }^{20}$ Department of Physics, Drexel University, Philadelphia, PA 19104, USA \\ ${ }^{21}$ Department of Astronomy, The Ohio State University, Columbus, OH 43210, USA \\ ${ }^{22}$ Enrico Fermi Institute, University of Chicago, Chicago, IL 60637, USA \\ ${ }^{23}$ Department of Astronomy, Case Western Reserve University, Cleveland, OH 44106, USA
}

2 November 2009

\begin{abstract}
The spectroscopic Sloan Digital Sky Survey (SDSS) Data Release 7 (DR7) galaxy sample represents the final set of galaxies observed using the original SDSS target selection criteria. We analyse the clustering of galaxies within this sample, including both the Luminous Red Galaxy (LRG) and Main samples, and also include the 2-degree Field Galaxy Redshift Survey (2dFGRS) data. In total, this sample comprises 893319 galaxies over $9100 \mathrm{deg}^{2}$. Baryon Acoustic Oscillations are observed in power spectra measured for different slices in redshift; this allows us to constrain the distance-redshift relation at multiple epochs. We achieve a distance measure at redshift $z=0.275$, of $r_{s}\left(z_{d}\right) / D_{V}(0.275)=0.1390 \pm 0.0037$ (2.7\% accuracy), where $r_{s}\left(z_{d}\right)$ is the comoving sound horizon at the baryon drag epoch, $D_{V}(z) \equiv\left[(1+z)^{2} D_{A}^{2} c z / H(z)\right]^{1 / 3}, D_{A}(z)$ is the angular diameter distance and $H(z)$ is the Hubble parameter. We find an almost independent constraint on the ratio of distances
\end{abstract}

\footnotetext{
^ E-mail: will.percival@port.ac.uk
}

(C) 0000 RAS 
$D_{V}(0.35) / D_{V}(0.2)=1.736 \pm 0.065$, which is consistent at the $1.1 \sigma$ level with the best fit $\Lambda$ CDM model obtained when combining our $z=0.275$ distance constraint with the WMAP 5 -year data. The offset is similar to that found in previous analyses of the SDSS DR5 sample, but the discrepancy is now of lower significance, a change caused by a revised error analysis and a change in the methodology adopted, as well as the addition of more data. Using WMAP5 constraints on $\Omega_{b} h^{2}$ and $\Omega_{c} h^{2}$, and combining our BAO distance measurements with those from the Union Supernova sample, places a tight constraint on $\Omega_{m}=0.286 \pm 0.018$ and $H_{0}=68.2 \pm 2.2 \mathrm{~km} \mathrm{~s}^{-1} \mathrm{Mpc}^{-1}$ that is robust to allowing $\Omega_{k} \neq 0$ and $w \neq-1$. This result is independent of the behaviour of dark energy at redshifts greater than those probed by the BAO and supernova measurements. Combining these data sets with the full WMAP5 likelihood constraints provides tight constraints on both $\Omega_{k}=-0.006 \pm 0.008$ and $w=-0.97 \pm 0.10$ for a constant dark energy equation of state.

Key words: cosmology: observations, distance scale, large-scale structure of Universe

\section{INTRODUCTION}

"What is the nature of dark energy?" is one of the current key questions in physical science. Distinguishing between competing theories will only be achieved with precise measurements of the cosmic expansion history and the growth of structure within it. Among current measurement techniques for the cosmic expansion, Baryon Acoustic Oscillations (BAO) appear to have the lowest level of systematic uncertainty (Albrecht et al. 2006).

$\mathrm{BAO}$ are a series of peaks and troughs, with a wavelength of approximately $0.06 \mathrm{~h} \mathrm{Mpc}^{-1}$ that are present in the power spectrum of matter fluctuations after the epoch of recombination, and on large-scales. They occur because the primordial cosmological perturbations excite sound waves in the relativistic plasma of the early universe (Silk 1968; Peebles \& Yu 1970; Sunyaev \& Zel'dovich 1970; Bond \& Efstathiou 1984, 1987; Holtzman 1989). Radiation pressure drives baryonic material away from the seed perturbations until the ionised material recombines at redshift $z \simeq 1000$. The momentum of the baryonic material means that the motion continues for a short time after recombination, until an epoch known as the baryon-drag epoch. The wavelength of the BAO is related to the comoving sound horizon at the baryon-drag epoch, which depends on the physical densities of matter $\Omega_{m} h^{2}$ and of baryons $\Omega_{b} h^{2}$ in the Universe. WMAP5 constraints on $\Omega_{b} h^{2}$ and $\Omega_{m} h^{2}$ Komatsu et al.2009) give that $r_{s}\left(z_{d}\right) \simeq 153.5 \mathrm{Mpc}$ (see Section7 for details).

BAO occur on relatively large scales, which are still predominantly in the linear regime at present day; it is therefore expected that BAO should also be seen in the galaxy distribution (Goldberg \& Strauss 1998; Meiksin et al. 1999; Springel et al. 2005; Seo \& Eisenstein 2005; White 2005; Eisenstein et al. 2007). We can therefore use BAO as standard rulers to constrain the expansion of the Universe if the comoving sound horizon at the baryon drag epoch is known. The apparent size of the BAO measured from observations then leads to measurements of the Hubble parameter and the angular diameter distance (Seo \& Eisenstein 2003; Bond \& Glazebrook 2003; Hu \& Haiman 2006; Matsubara 2004).

The acoustic signature has now been convincingly detected at low redshift (Percival et al. 2001; Cole et al. 2005; Eisenstein et al. 2005; Huetsi 2006) using the 2dF Galaxy Redshift Survey (2dFGRS; Colless et al. 2003) and the Sloan Digital Sky Survey (SDSS; York et al. 2000). The detection has subsequently been refined using more data and better techniques, and is now producing competitive constraints on cosmological models. Tegmark et al. (2006) analysed the Sloan Digital Sky Survey (SDSS) Data Release
4 (DR4; Adelman-McCarthy et al. 2006) Luminous Red Galaxy (LRG) sample. Percival et al. (2007a b) presented the power spectrum of the Sloan Digital Sky Survey (SDSS) Data Release 5 (DR5; Adelman-McCarthy et al. 2007) galaxy sample and considered the shape of the power spectrum and measured the matter density using the BAO features. Percival et al. (2007c) took this analysis a stage further by fitting the SDSS data, combined with the 2dFGRS, with models of the distance-redshift relation. Gaztanaga et al. (2008) and Sanchez et al. (2009) have also analysed the SDSS DR6 (Adelman-McCarthy et al. 2008) sample, obtaining cosmological constraints from the radial and spherically averaged BAO signal. In a recent analysis, Kazin et al. (2009) have calculated the correlation function of the SDSS DR7 (Abazajian et al. 2009) LRG sample, and have shown that their results agree with those presented in our paper. Two studies have also considered the clustering of the LRGs at high redshift within the SDSS survey, using photometric redshifts to estimate galaxy distances (Padmanabhan et al. 2007; Blake et al. 2007).

In this paper, we analyse the clustering of galaxies in the spectroscopic SDSS DR7 sample, including both LRG and Main galaxy samples, combined with the 2dFGRS, and measure the BAO signal in a series of redshift slices. SDSS DR7 marks the final release of galaxies observed using the standard SDSS targeting algorithm, and the sample we analyse covers a solid angle of $7930 \mathrm{deg}^{2}$, including a $7190 \mathrm{deg}^{2}$ contiguous region in the North Galactic Cap. The Baryon Oscillation Spectroscopic Survey (BOSS; Schlegel et al. 2009a), part of the SDSS-III project, will adopt a different targeting algorithm, focusing on galaxies and quasars at higher redshifts.

The observed amplitude of the large-scale galaxy clustering depends on both galaxy colour and luminosity (Tegmark et al. 2004; Zehavi et al. 2005; (Swanson et al. 2008). Using the SDSS DR5 sample, Cresswell \& Percival (2009) showed that for blue galaxies, the deviation in the shape of the galaxy power spectrum from the linear matter power spectrum at $k>0.1 h \mathrm{Mpc}^{-1}$ is a strong function of luminosity, while it is almost constant for red galaxies. It is therefore difficult to extract the underlying matter power spectrum from a galaxy power spectrum measured for a population of galaxies where the distribution of galaxy colours and luminosities changes with spatial location, such as that provided by a magnitude-limited catalogue. In contrast, the luminous red galaxy population, which comprises the high redshift part of the sample analysed here, has a simpler relation with the matter field, in that there is a single galaxy population to consider (Reid et al. 2008). 
In a companion paper (Reid et al. 2009), we apply a grouping algorithm to recover the halo power spectrum from the LRGs, then calibrate the relation of the halo power spectrum to the linear theory power spectrum using simulations. We are then able to extract cosmological information from the large scale shape of the power spectrum in addition to the BAO signal, though the constraints are more tightly embedded in the assumed cosmological framework.

BAO in the galaxy power spectrum are only weakly affected by the effects of non-linear structure formation and scaledependent galaxy bias, because they are on such large scales. The primary consequence is a damping on small scales, which can be well approximated by a Gaussian smoothing (Bharadwai 1996; Crocce \& Scoccimarro 2006, 2008; Eisenstein et al. 2007; Matsubara 2008a b). The observed BAO, defined as the ratio of the observed power spectrum $P_{\mathrm{obs}}$ to a smooth fit to this power $P_{\mathrm{nw}}$, $B A O_{\text {obs }} \equiv P_{\text {obs }} / P_{\text {nw }}$, are related to the original BAO in the linear matter power spectrum $B A O_{\text {lin }}$, defined similarly, by

$B A O_{\mathrm{obs}}=G_{\mathrm{damp}} B A O_{\mathrm{lin}}+\left(1-G_{\mathrm{damp}}\right)$,

where $G_{\text {damp }}=\exp \left(-\frac{1}{2} k^{2} D_{\text {damp }}^{2}\right)$, and the damping scale, $D_{\text {damp }}$ is set to $10 \mathrm{~h}^{-1} \mathrm{Mpc}$ for redshift-space power spectra at $z \simeq 0.3$ (Eisenstein et al. 2007). This damping of the linear power is a relatively benign effect as it does not affect the positions of the BAO, although it does reduce the signal available. Additional, more pernicious effects such as the mixing of modes in the power spectrum, can generate shifts in the BAO position (Crocce \& Scoccimarro 2008); for biased tracers, these offsets can be at the percent level (Smith et al. 2007), and are therefore important as we wish to make percent level distance measurements.

In our analysis, we measure BAO relative to a model that allows for smooth changes in the underlying shape of the power spectrum, which alleviates some of this shift. Physical models of BAO positions in observed redshift-space power spectra relative to a such a fitted smooth model (Crocce \& Scoccimarro 2008; Smith et al. 2008; Sanchez et al. 2009; Padmanabhan \& White 2009), and numerical simulations (Angulo et al. 2008; Seo et al. 2008; Kim et al. 2009) suggest we should expect residual shifts at the sub-percent level. These are below the precision of current experiments: e.g. in this paper we present a BAO distance scale measurement with $2.7 \%$ accuracy. Therefore, we adopt a procedure that allows for the damping as well as smooth changes in the underlying shape of the power spectrum, but no more. The analysis of future surveys, which will lead to tighter distance-redshift constraints, will clearly also have to allow for non-linear effects, either by physical modeling, simulations, or by using methods which attempt to reconstruct the initial fluctuation field (Eisenstein et al. 2007; Seo et al. 2008; Padmanabhan et al 2009).

The SDSS and 2dFGRS data are discussed in Sections 2 and 3 The basic methodology, presented in Section 4 is similar to that of Percival et al. (2007c), although we have revised the calculation of the window function to increase the computational speed. We also perform an extensive test of the derived errors, running mock catalogues through our full analysis pipeline to test the confidence intervals quoted (Section 5). Results are presented in Section 6 and 7 tested for robustness in Section 8 and placed in a cosmological context in Sections and 9 A comparison with our DR5 analyses is given in Section 10 and we finish with a discussion in Section 11

In this paper we use the standard cosmological parameters. For flat $\Lambda \mathrm{CDM}$ models these are the Hubble constant $H_{0}$, the densities of baryonic matter $\Omega_{b}$, cold dark matter $\Omega_{c}$, all matter $\Omega_{m}$, and dark energy $\Omega_{\Lambda}$. Going beyond this simple class of models, we use the equation of state of the dark energy $w$, the curvature energy density
$\Omega_{k}$ and total energy density $\Omega_{\text {tot }}$. When combining with information from the CMB, we also consider some parameters that are not constrained by the BAO: $\tau$ is the optical depth to re-ionization, $n_{s}$ is the scalar spectral index, and $A_{05}$ is the amplitude of curvature perturbations at $k=0.05 \mathrm{Mpc}^{-1}$.

\section{THE DATA}

The SDSS-I and SDSS-II projects used a $2.5 \mathrm{~m}$ telescope (Gunn et al.2006), to obtain imaging data in five passbands $u, g, r$, $i$ and $z$ (Fukugita et al. 1996; Gunn et al.|1998). The images were reduced (Lupton et al.2001; Stoughton et al.2002; Pier et al.2003; Ivezic et al. 2004) and calibrated (Lupton et al. 1999; Hogg et al. 2001; Smith et al. 2002; ;ucker et al. 2006), and galaxies were selected in two ways for follow-up spectroscopy. The main galaxy sample (Strauss et al. 2002) targeted galaxies brighter than $r=$ 17.77 (approximately 90 per square degree, with a weighted median redshift $z=0.10$ ). The DR7 sample (Abazajian et al. 2009) used in our analysis includes 669905 main galaxies (Strauss et al. 2002) with a median redshift of $z=0.12$, selected to a limiting Galactic extinction-corrected Petrosian magnitude $r<17.77$, or $r<17.5$ in a small subset of the early data from the survey. The effect of the inclusion of the early SDSS data is tested in Section 8.2 In addition, our sample includes 80046 Luminous Red Galaxies (LRGs; Eisenstein et al. 2001), which form an extension of the SDSS spectroscopic survey to higher redshifts $0.2<z<0.5$. Of the main galaxies, 30530 are also classified as LRGs and are intrinsically luminous with $\mathrm{M}_{0.1_{r}}<-21.8$, where $\mathrm{M}_{0.1_{r}}$ is the Galactic extinction and K-corrected $r$-band absolute galaxy magnitude. We apply this requirement to all of our LRGs, so our sample includes 110576 LRGs in total, with a weighted median redshift of $z=0.31$. Although the main galaxy sample contains significantly more galaxies than the LRG sample, the LRG sample covers more volume. Redshift distributions for these samples are shown in figure 2 of Percival et al. (2007b). In our default analysis we use SDSS Petrosian magnitudes calibrated using the "uber-calibration" method (Padmanabhan et al 2008), although we test against data calculated using the original calibration methodology (Tucker et al. 2006). Where specified, we have K-corrected the galaxy luminosities using the methodology outlined by Blanton et al. (2003a b). Further details of the cuts applied to the data can be found in Percival et al. (2007b).

Due to the finite size of the fibers, spectra cannot be obtained for both objects in a pair closer than 55 arcsec, within a single spectroscopic tile. Tiling (Blanton et al. 2003a) deals with this to some extent by allowing plate overlaps to provide multiple observations of crowded regions. Even so, not all galaxies in such regions which meet the target selection criteria could be observed. Zehavi et al. (2002) corrected for this undersampling by assigning the redshift of the nearest observed galaxy to a galaxy which was not observed due to crowding, and showed that this provides sufficient correction for large-scale structure studies. We apply this correction in the present work, and test it to show that our results are insensitive to this in Section 8.1

In order to increase the volume covered at redshift $z<0.3$, we include 143368 galaxies from the 2dFGRS sample. These galaxies, selected to an extinction-corrected magnitude limit of approximately $b_{J}=19.45$ (Colless et al. 2003) from regions of sky not covered by the SDSS sample, cover two contiguous regions totalling $\sim 1200 \mathrm{deg}^{2}$. They do not include the 2 dFGRS random fields, a set of 99 random 2 degree fields spread over the full south- 


\begin{tabular}{cccrrr}
\hline SLICE & $z_{\min }$ & $z_{\max }$ & $N_{\text {gal }}$ & $V_{\text {eff }}$ & $\bar{n}$ \\
\hline 1 & 0.0 & 0.5 & 895834 & 0.42 & 128.1 \\
2 & 0.0 & 0.4 & 874330 & 0.38 & 131.2 \\
3 & 0.0 & 0.3 & 827760 & 0.27 & 138.3 \\
4 & 0.1 & 0.5 & 505355 & 0.40 & 34.5 \\
5 & 0.1 & 0.4 & 483851 & 0.36 & 35.9 \\
6 & 0.2 & 0.5 & 129045 & 0.27 & 1.92 \\
7 & 0.3 & 0.5 & 68074 & 0.15 & 0.67 \\
\hline
\end{tabular}

Table 1. Parameters of the redshift intervals analysed. $V_{\text {eff }}$ is given in units of $h^{-3} \mathrm{Gpc}^{3}$, and was calculated as in Eq. (2) using an effective power spectrum amplitude of $\bar{P}=10^{4} h^{-3} \mathrm{Mpc}$, appropriate on scales $k \sim 0.15 h \mathrm{Mpc}^{-1}$ for a population with bias $b=1.7$. The average galaxy number density in each bin $\bar{n}$ is in units of $10^{-4}\left(h^{-1} \mathrm{Mpc}\right)^{3}$.

ern Galactic cap, as these would complicate the window function. The galaxies cover $0<z<0.3$, with a weighted median at $z=0.17$. The redshift distribution of the sample was analysed as in Cole et al. (2005) for $0<z<0.3$, and we use the same synthetic catalogues to model the unclustered expected galaxy distribution within the reduced sample.

We assume that each galaxy is biased with a linear deterministic bias model, and that this bias depends on $\mathrm{M}_{0.1_{r}}$ according to Tegmark et al. (2004) and Zehavi et al. (2005). All galaxies were weighted using this model so the fluctuation amplitudes match those of $L_{*}$ galaxies, where $L_{*}$ was calculated separately for the SDSS and 2dFGRS. We include an extra normalisation factor to the 2dFGRS galaxy bias model to correct the relative bias of $L_{*}$ galaxies in the different surveys. This was calculated by matching the normalisation of the 2dFGRS and SDSS bias-corrected power spectra for $k<0.1 h \mathrm{Mpc}^{-1}$. In principle, we could have added information on galaxy bias from the BAO, since the small-scale damping (see Eq. 1) depends on how strongly nonlinear the underlying dark matter density fluctuations are. As we show in Section 8.6 this information is limited for the current data, but future surveys may be able to exploit changes in this damping as a function of galaxy properties, such as colour and luminosity.

\section{SPLITTING INTO SUB-SAMPLES}

In order to probe the distance-redshift relation in detail, ideally we would analyze BAO measured in many independent redshift slices. However, if the slices are too narrow in redshift, then there is insufficient signal and the BAO cannot be recovered with sufficient accuracy to give a likelihood with close to a Gaussian distribution (see the discussion in Section 5]. If the slices are too wide, or too many overlapping slices are chosen, the covariance matrix becomes close to singular, potentially leading to numerical instability. In order to balance these competing requirements, we have chosen to analyse the redshift slices presented in Table 1 The power spectra will be correlated, and these correlations, together with correlations of $P(k)$ values at different $k$ within each redshift slice, are included in the covariance matrices in our analysis. Note that we include slice 7, for which the effective volume is relatively small, because of the interesting redshift range covered.

As well as giving the redshift limits of the slices in Table 1 we also give the number of galaxies in each including both the $2 \mathrm{dF}$ GRS and the SDSS, and the effective volume, calculated from the integral (Feldman et al. 1994)
$V_{\text {eff }}=\int d^{3} r\left[\frac{\bar{n}(\mathbf{r}) \bar{P}}{1+\bar{n}(\mathbf{r}) \bar{P}}\right]^{2}$,

where $\bar{n}(\mathbf{r})$ is the observed comoving number density of the sample at location $\mathbf{r}$ and $\bar{P}$ is the expected power spectrum amplitude. To calculate $V_{\text {eff }}$ for our redshift slices, distances were calculated assuming a fiducial flat $\Lambda$ CDM cosmology with $\Omega_{m}=0.25$. For the numbers given in Table 1, we fix $\bar{P}=10^{4} h^{-3} \mathrm{Mpc}^{3}$, appropriate on scales $k \sim 0.15 \mathrm{~h} \mathrm{Mpc}^{-1}$ for a population with bias $b=1.7$. For comparison, Eisenstein et al. (2005) analyse a sample with $V_{\text {eff }}=0.13 h^{-3} \mathrm{Gpc}^{3}$, approximately a third of the effective volume of slice 1 .

We fit models to three sets of power spectra:

(i) We fit a single power spectrum for the SDSS LRG sample covering $0.15<z<0.5$.

(ii) We fit three power spectra for slices 1,3 and 6 approximately corresponding to the procedure adopted by Percival et al. (2007c). Although we now use slices constrained by redshift rather than galaxy type, the $0<z<0.3$ slice is dominated by SDSS main galaxies, while the $0.2<z<0.5$ slice is dominated by LRGs.

(iii) We fit six power spectra for slices $2 \rightarrow 7$, which allows a test of the distance-redshift relation at greater resolution.

We consider option (i) to tie in with the analysis presented by Reid et al. (2009), and to demonstrate the effect of collapsing the clusters in redshift-space where we try to reconstruct the halo power spectrum. Option (ii) is close to the approach of Percival et al. (2007c), where the SDSS main galaxies and 2dFGRS galaxies were analysed separately from the SDSS LRGs. Option (iii) allows us to see if there is more information available beyond measuring the distance-redshift relation at two redshifts. The slices do overlap in redshift, but we will properly take into account the covariance between the results when we fit to cosmological parameters.

\section{BASIC METHODOLOGY}

Power spectra were calculated for each catalogue using the Fourier method of Feldman et al. (1994), as applied by Percival et al. (2007b). In this method a weighted galaxy over-density field is defined and Fourier transformed, then the spherically averaged power is measured. We use the luminosity dependent galaxy weights advocated by Percival et al. (2004), as described in Section 2 To construct the over-density field, we need to quantify the expected spatial distribution of galaxies, in the absence of clustering. The standard method for this is to use an unclustered random catalogue, which matches the galaxy selection. To calculate this random catalogue, we fitted the redshift distributions of the galaxy samples with a spline fit (Press et al. 1992), and the angular mask was determined using a routine based on a HEALPIX (Górski et al. 2005) equalarea pixelization of the sphere (Percival et al. 2007b). Percival et al. (2007b) used a random catalogue containing ten times as many points as galaxies. For the sparse LRGs, this approach induces significant shot noise, so we now use one hundred times as many random points as LRGs. We have also increased the resolution at which the radial distribution of galaxies is quantified, now using a spline fit (Press et al. 1992) with nodes separated by $\Delta z=0.0025$. As an alternative to this radial selection, we could have simply adopted the redshift of a randomly chosen galaxy for each of our points in the random catalogue. In Section 8.7 we show that these two possibilities give consistent results. 
Galaxy redshifts were converted to distances using a fiducial cosmology (flat $\Lambda$ CDM model with $\Omega_{m}=0.25$ ). For each distance-redshift model to be tested, we do not recalculate the power spectrum, but instead change the interpretation of the power spectrum computed assuming the fiducial $\Lambda$ CDM galaxy distances. We do this through a window function, which relates the true and measured power spectra. This follows the procedure adopted by Percival et al. (2007c), but we now use a revised, computationally less intensive method for calculating the windows, as described in Appendix A

A model of the BAO was created by fitting a linear matter power spectrum, calculated using CAMB (Lewis et al. 2000), which numerically solves the Boltzman equation describing the physical processes in the Universe before the baryon-drag epoch, with a cubic spline to remove the broad shape of the power, leaving the oscillations. The theoretical BAO were then damped with a Gaussian model as in Eq. (1), following the simulation results of Eisenstein et al. (2007). For our default fits, we assume that the damping scale $D_{\text {damp }}=10 h^{-1} \mathrm{Mpc}$ (Eisenstein et al. 2007), but we also consider fits where this scale is varied (Section 8.6. As discussed in Section 1 we do not attempt to correct for any shift induced by non-linear physics, because they are expected to be at a level below our statistical error.

The power spectrum measured from the data was fitted by a model constructed by multiplying this BAO model with a cubic spline (Press et al. 1992), which enables the power spectrum model to match the overall shape of the data power spectrum. Each power spectrum model was then convolved with a window function that corrects for both the survey geometry and the differences between our fiducial cosmological model used to convert redshift to distances and the cosmological model to be tested (see Appendix A. The free parameters of the model are the nine nodes of the cubic spline fixed empirically at $k=0.001$, and $0.025 \leqslant k \leqslant 0.375$ with $\Delta k=0.05$, and the parametrisation of $D_{V}(z)$ used to calculate the correct window function. The spline nodes were refitted for every cosmology (or $D_{V}(z)$ ) tested. A power spectrum model with this spline node separation was tested by fitting many mock power spectra by Percival et al. (2007a) and was shown to match these without leaving significant residuals in the measured "shift" between BAO in the model and data power spectra. This approach was also considered by Sanchez et al. (2008), who found that it did not induce a bias in the recovered BAO constraints.

For a redshift survey in a thin shell, the position of the BAO approximately constrains $d_{z} \equiv r_{s}\left(z_{d}\right) / D_{V}(z)$, where $r_{s}\left(z_{d}\right)$ is the comoving sound horizon at the baryon drag epoch, $D_{V}(z) \equiv$ $\left[(1+z)^{2} D_{A}^{2} c z / H(z)\right]^{1 / 3}$ Eisenstein et al. 2005; Percival et al. 2007c), $D_{A}$ is the angular diameter distance, and $H(z)$ is the Hubble parameter. We see that, although our power spectrum fitting procedure measures $D_{V}(z)$ for a fixed BAO model, we should consider the constraints as measurements of $d_{z}$, with $r_{s}\left(z_{d}\right)$ calculated for the flat $\Lambda \mathrm{CDM}$ model for which we created the BAO model, $r_{s}\left(z_{d}\right)=111.4 h^{-1} \mathrm{Mpc}=154.7 \mathrm{Mpc}$, using equation 6 of Eisenstein \& Hu (1998), and assuming $h=0.72, \Omega_{b} h^{2}=0.0223$, and $\Omega_{m}=0.25$. This value of $r_{s}\left(z_{d}\right)$ is only used to index this model: as described above, the actual BAO model was calculated from a power spectrum predicted by CAMB. If the constraints provided in this paper are to be used to constrain a set of models where $r_{s}\left(z_{d}\right)$ for this fiducial model is calculated in a different way (i.e. not using equation 6 of Eisenstein \& Hu 1998), then our constraints should be adjusted to match.

The comoving distance-redshift relation is modelled as a cubic spline in the parameter $D_{V}(z)$. We consider models for $D_{V}(z)$

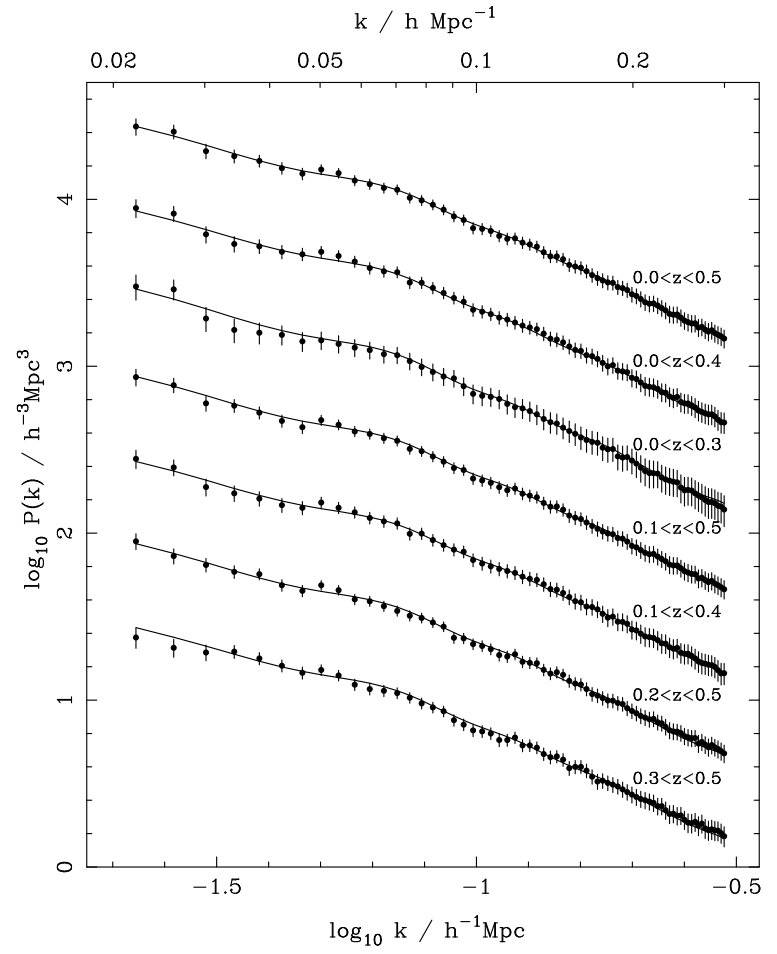

Figure 1. Average power spectra recovered from the Log-Normal catalogues (solid lines) compared with the data power spectra (solid circles with 1- $\sigma$ errors) for the six samples in Table 1 The errors on the data were calculated from the diagonal elements of the covariance matrix calculated from these log-normal catalogues. The power spectra have been offset by $0.5 \mathrm{dex}$ for clarity, with the upper power spectrum having the correct normalisation.

with two nodes at $z=0.2$ and $z=0.35$, or with four nodes at $z=0.1, z=0.2, z=0.3, z=0.45$. Results are presented as constraints on $d_{z}$. The error between cubic spline fits to $D_{V}(z)$ with two nodes at $z=0.2$ and $z=0.35$, to the $\Lambda$ CDM distanceredshift relations was shown in figure 1 of Percival et al. (2007c), and is $<1 \%$ for a flat $\Lambda$ CDM cosmology with $\Omega_{m}=0.25$ at $z \geqslant 0.15$.

Power spectra are presented for the redshift slices described in Section 3 in Fig. 1 for 70 band powers equally spaced in $0.02<$ $k<0.3 h \mathrm{Mpc}^{-1}$. We see that the power spectra from the different redshift intervals are remarkably consistent, with $P(k)$ decreasing almost monotonically to small scales.

In order to calculate the covariances between the data, we have created 10000 Log-Normal (LN) density fields (Coles \& Jones 1991; Cole et al. 2005) from which we have drawn overlapping catalogues for each of our 7 redshift slices. Catalogues were calculated on a $(512)^{3}$ grid with box length $4000 h^{-1} \mathrm{Mpc}$. Unlike N-body simulations, these mock catalogues do not model the growth of structure, but instead return a density field with a log-normal distribution, similar to that seen in the real data. The window functions for these catalogues were matched to that of the 2dFGRS+SDSS catalogue with the original calibration. The input power spectrum was a cubic spline fit matched to the data power spectra (i.e. the smooth part of our standard model), multiplied by our default damped $\Lambda$ CDM BAO model calculated using CAMB (Lewis et al. 2000). The $\mathrm{LN}$ power spectra were used to determine a covariance matrix between slices and for different band powers in each slice, assuming that the band-powers were drawn from a multi-variate Gaussian distribution. Average recovered power spectra for each 
redshift interval are compared with the data power spectra in Fig. 1 Clearly the general shape of the average power spectra of the LN catalogues is well matched to that recovered from the data. Using the inverse of this covariance matrix, we estimate the likelihood of each model assuming that the power spectra band-powers for $0.02<k<0.3 h \mathrm{Mpc}^{-1}$ were drawn from a multi-variate Gaussian distribution.

\section{TESTING THE ANALYSIS METHOD WITH MOCK DATA}

\subsection{The model fit}

We now consider using a subset of our LN catalogues to test our analysis procedure. For 1000 of the mock catalogues, we fit spline $\times \mathrm{BAO}$ models to extract distance constraints from the $\mathrm{BAO}$, as described in Section 4 A small average shift of $1.3 \%$ in the BAO scale was recovered between the power recovered from the LN catalogues, and the input power spectrum used to create them. If we correct the 1000 power spectra measured from the LN catalogues by multiplying each power spectrum by the expected power divided by the average recovered power spectrum, the average shift drops below $0.3 \%$, well within $1 \sigma$.

To test the origin of the observed $1.3 \%$ shift, we have also drawn 1000 power spectrum realisations from a multi-variate Gaussian distribution with covariance and mean matched to those of the data. These mock catalogues were fitted using the procedure described in Section 4 No shift in the BAO position was found from the fits to these catalogues, within the statistical limits of the analysis $(\sim 0.3 \%)$. The distribution of recovered distance constraints was well matched to that recovered from fitting the corrected LN power spectra. Thus the $1.3 \%$ shift described above must be due to the $\mathrm{LN}$ procedure itself. The expected shift is dependent on the statistic used to measure the BAO position. The Log-Normal correlation function $\xi_{L N}$, and Gaussian correlation function $\xi_{G}(r)$ of a field with the same power spectrum but with Gaussian statistics, are related by $1+\xi_{L N}(r)=\exp \left[\xi_{G}(r)\right]$. If we had used the peak in the correlation function as our standard ruler then, for the LN catalogues, we would have expected no BAO shift. However, the same is not true of our $\mathrm{BAO} \times$ spline model fitting procedure, which fits the $\mathrm{BAO}$ in the power spectrum over a range of scales.

Numerical simulations offer a better way to model the true Universe, and recent results from simulations show that we should expect a less significant shift between the BAO positions in the linear matter and galaxy power spectra than the $1.3 \%$ shift found for the LN catalogues (Seo \& Eisenstein 2003; Springel et al. 2005; Seo \& Eisenstein 2007; Angulo et al. 2008). The exact shift required for the catalogues we analyse is not well constrained by these simulation results, and we consequently do not alter our analysis to include such a shift.

\subsection{The likelihood surface}

We use the Gaussian and LN power spectra samples to assess the nature of the likelihood for the BAO scale recovery. We consider fits to either three or six power spectra as described in Section 3 . parametrising $D_{V}(z)$ with a cubic spline with two non-zero nodes at $z=0.2$ and $z=0.35$. For each of the 1000 fits, we have measured the difference between the maximum likelihood value and the likelihood at the parameters of the true cosmological model.

\begin{tabular}{ccccc}
\hline$-2 \ln \mathcal{L} / \mathcal{L}_{\text {true }}$ & \multicolumn{4}{c}{ fraction of samples } \\
& \multicolumn{2}{c}{3 slices } & \multicolumn{2}{c}{6 slices } \\
& standard & revised & standard & revised \\
\hline$<2.3$ & 0.579 & 0.666 & 0.551 & 0.667 \\
$<6.0$ & 0.892 & 0.946 & 0.862 & 0.948 \\
$<9.3$ & 0.966 & 0.983 & 0.955 & 0.981 \\
\hline
\end{tabular}

Table 2. Fraction of fits to the Log-Normal power spectra in which the ratio of the likelihood maximum and the likelihood for the true cosmological model is less than the given limit. For a Gaussian likelihood, these limits correspond to $68 \%, 95 \%$ and $99 \%$ confidence intervals. We show results where we have corrected the errors as described in the text by multiplying the band-power errors by 1.14 for three redshift slices, and 1.21 for six redshift slices.

The fraction of samples with $-2 \ln \mathcal{L} / \mathcal{L}_{\text {true }}<2.3,6.0,9.3$, corresponding to $68 \%, 95 \%$ and $99 \%$ confidence intervals, are given in Table 2 We find that in order to match the expected numbers of samples with likelihoods within the standard $1 \sigma$ Gaussian confidence intervals, we must increase the errors on the power spectrum band powers by $14 \pm 2 \%$ if we fit to three power spectra. For fits to six power spectra, we must increase the errors by $21 \pm 2 \%$ in order to match the expected $1 \sigma$ Gaussian confidence intervals. Although in this paper we do not consider fitting to a single power spectrum, we have repeated this analysis for BAO fits to the LRG sample of Reid et al. (2009), and find that we must increase the errors on the power spectrum band powers by $10 \pm 2 \%$ to match the expected confidence intervals.

Because the same increase in the confidence intervals is required for both LN and Gaussian mock catalogues, this change must be caused by the methodology of fitting BAO, rather than the Gaussian to Log-Normal density field transition. In fact, we believe that it is caused by the non-Gaussian nature of the likelihood surface. We should expect the likelihood surface to be non-Gaussian to some extent in any case because there is a minimum in the likelihood where the observed and model BAO are perfectly out of phase in $k$-space: this represents the worst possible match between data and model. Adjusting the covariance matrix to match the distribution of best-fit distance-scales to the expected $68 \%$ confidence interval does not quite match the $95 \%$ or $99 \%$ confidence intervals, although it corrects for most of the difference. This shows that the confidence intervals cannot perfectly match those for a Gaussian distribution.

To test this further, we have created a set of 1000 Gaussian power spectrum realisations with errors that are $10 \%$ of those in our standard sample. For these catalogues, the distribution of best-fit $D_{V}(z)$ matches that expected from the likelihood distribution under Gaussian assumptions. No correction is required, and the likelihood distribution is much closer to that for a multi-variate Gaussian distribution around the likelihood maximum. Thus the requirement to increase the errors on the data disappears when we fit less noisy data, as we would expect if it is caused by fitting noisy data, which is giving a non-Gaussian likelihood surface.

The average likelihood surfaces measured from our 1000 fits to sets of three power spectra and six power spectra drawn from LN catalogues are shown in Fig. 2. We also plot the centre of the local likelihood maxima nearest to the input cosmological parameters for each model. The fractions of points within each contour are given in Table 2. the errors on the power spectrum band powers have been adjusted for each plot as described above so that $\sim 68 \%$ of the points lie within the $-2 \ln \mathcal{L}=2.3$ contour. 

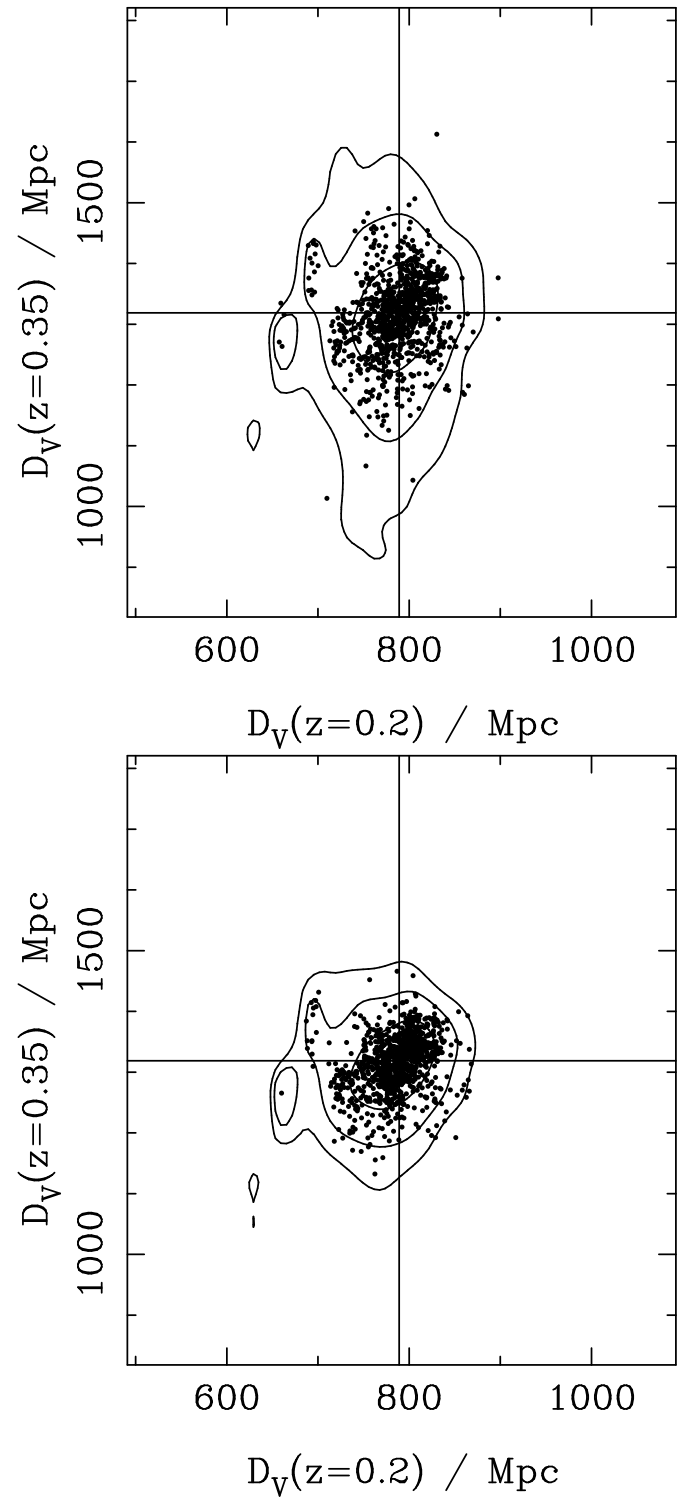

Figure 2. Average likelihood contours recovered from the analysis of three power spectra (top panel) and six power spectra (bottom panel) measured from 1000 Log-Normal density fields. Contours are plotted for $-2 \ln \mathcal{L}=$ $2.3,6.0,9.2$, corresponding to two-parameter confidence of $68 \%, 95 \%$ and 99\% for a Gaussian distribution. Contours were calculated after increasing the errors on the power spectrum band-powers as described in the text. Solid circles mark the locations of the likelihood maxima closest to the true cosmology. We have plotted the likelihood surface as a function of $D_{V}(z) / \mathrm{Mpc}$, for fixed $r_{s}\left(z_{d}\right)=154.7 \mathrm{Mpc}$, to show distance errors if the comoving sound horizon is known perfectly. The values of $D_{V}$ for our input cosmology are shown by the vertical and horizontal solid lines.

\section{RESULTS}

Baryon Acoustic Oscillations are observed in the power spectra recovered from all redshift slices of the SDSS+2dFGRS sample described in Section 3 , and are shown in Fig. 3. where we plot the measured power spectra divided by the spline component of the best-fit model. In our default analysis we fit power spectra from six redshift slices as described in Section 3 using a spline for $D_{V}(z)$ with two nodes at $z=0.2$ and $z=0.35$. We assume a fixed BAO damping scale of $D_{\text {damp }}=10 h^{-1} \mathrm{Mpc}$ and fit to all SDSS and non-overlapping 2dFGRS data. The effect of

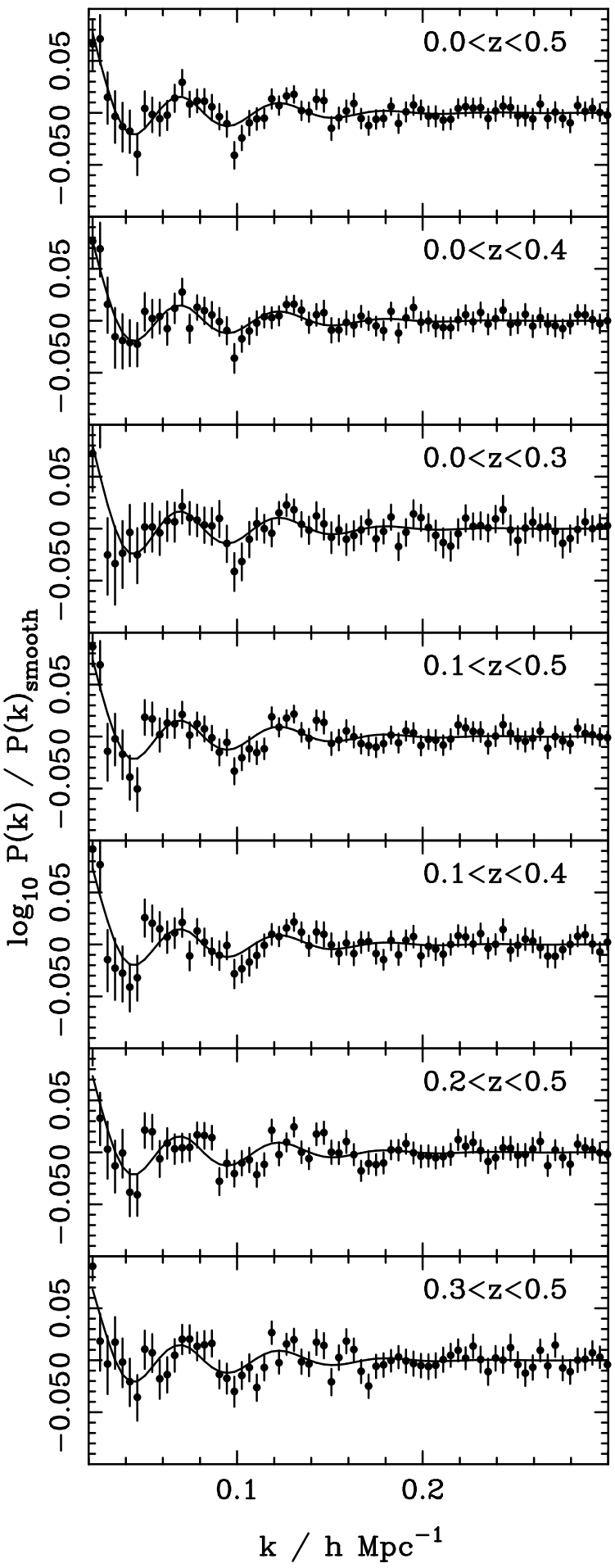

Figure 3. BAO recovered from the data for each of the redshifts slices (solid circles with 1- $\sigma$ errors). These are compared with BAO in our default $\Lambda$ CDM model (solid lines).

these assumptions is considered in Section 8 The resulting likelihood surface is shown in Fig. 4 as a function of $D_{V}(z) / \mathrm{Mpc}$, for fixed $r_{s}\left(z_{d}\right)=154.7 \mathrm{Mpc}$, to show distance errors if the comoving sound horizon is known perfectly. The constraints should be considered measurements of $r_{s}\left(z_{d}\right) / D_{V}(z)$ (see Section 4 . Fig. 4 reveals a dominant likelihood maximum close to the parameters of a $\Lambda$ CDM cosmology with $\Omega_{m}=0.25, h=0.72, \&$ 


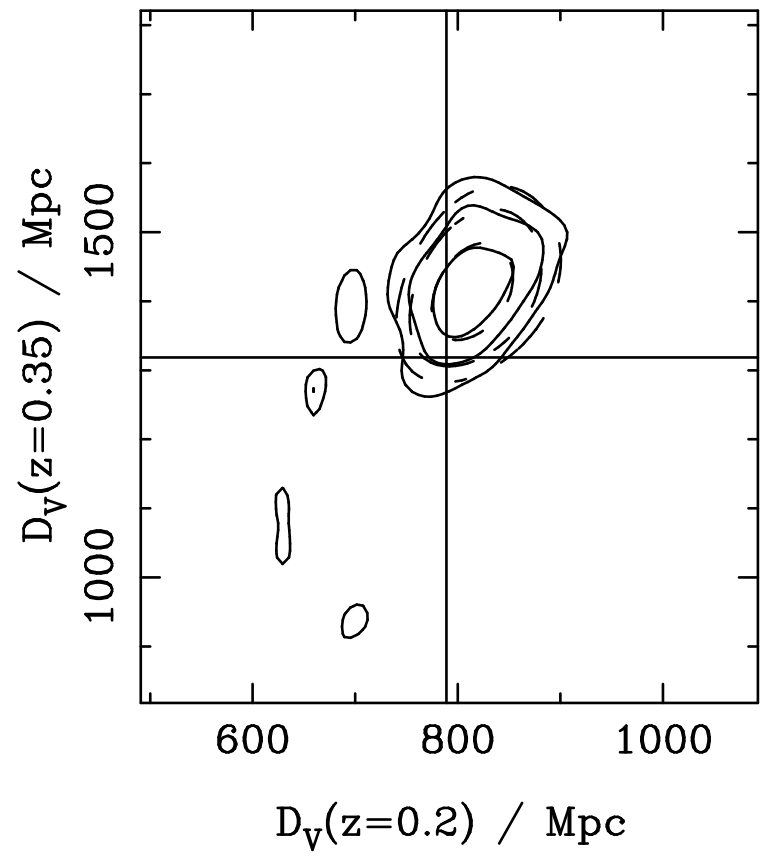

Figure 4. Likelihood contour plots for fits of two $D_{V}(z)$ cubic spline nodes at $z=0.2$ and $z=0.35$, calculated for our default analysis using six power spectra, uber-calibration, a fixed BAO damping scale of $D_{\text {damp }}=10 h^{-1} \mathrm{Mpc}$, and for all SDSS and non-overlapping 2dFGRS data. Solid contours are plotted for $-2 \ln \mathcal{L} / \mathcal{L}_{\text {true }}<2.3,6.0,9.3$, which for a multi-variate Gaussian distribution with two degrees of freedom correspond to $68 \%, 95 \%$ and $99 \%$ confidence intervals. Likelihoods were adjusted to match these Gaussian confidence intervals as described in Section 5 We have plotted the likelihood surface as a function of $D_{V}(z) / \mathrm{Mpc}$, for fixed $r_{s}\left(z_{d}\right)=154.7 \mathrm{Mpc}$, to show distance errors if the comoving sound horizon is known perfectly. We also show a multi-variate Gaussian fit to this likelihood surface (dashed contours). The values of $D_{V}$ for a flat $\Lambda$ CDM cosmology with $\Omega_{m}=0.25, h=0.72, \& \Omega_{b} h^{2}=0.0223$ are shown by the vertical and horizontal solid lines.

$\Omega_{b} h^{2}=0.0223$. There are also weaker secondary maxima at lower $D_{V}(0.2)$, which are considered further in Section 8.8 The significance of detection of BAO corresponds to $\Delta \chi^{2}=13.1$, which is approximately $3.6 \sigma$. As this is relative to an arbitrary smooth model, this test is more general, and hence the significance cannot be directly compared with results presented by Eisenstein et al. (2005).

We have matched the likelihood surface shown in Fig. 4 around the dominant maximum to a multi-variate Gaussian model. Using this Gaussian fit, we find that the best fit model has

$$
\begin{aligned}
d_{0.2} & =0.1905 \pm 0.0061(3.2 \%), \\
d_{0.35} & =0.1097 \pm 0.0036(3.3 \%),
\end{aligned}
$$

where $d_{z} \equiv r_{s}\left(z_{d}\right) / D_{V}(z)$. These results are correlated with correlation coefficient $r=0.337$. For a cosmological distanceredshift model with $\hat{d}_{z}$ the likelihood can be well approximated by a multi-variate Gaussian with covariance matrix

$C \equiv\left(\begin{array}{cc}\left\langle\Delta d_{0.2} \Delta d_{0.2}\right\rangle & \left\langle\Delta d_{0.2} \Delta d_{0.35}\right\rangle \\ \left\langle\Delta d_{0.35} \Delta d_{0.2}\right\rangle & \left\langle\Delta d_{0.35} \Delta d_{0.35}\right\rangle\end{array}\right)$

where $\Delta d_{z} \equiv d_{z}-\hat{d}_{z} . C$ has inverse

$C^{-1}=\left(\begin{array}{cc}30124 & -17227 \\ -17227 & 86977\end{array}\right)$.
Without correcting the covariance matrix using the results from fitting to the LN power spectra as described in Section 5 the original average errors on $d_{0.2}$, and $d_{0.35}$ were 0.0051 and 0.0029 , $16 \%$ and $24 \%$ lower than those in Eq. (3), respectively. Compare with the band power errors which were increased by $21 \%$, and we see that there is not a direct relation between changes in the band power errors and errors on $d_{z}$, because of the non-linear nature of the fit.

We diagonalise the covariance matrix of $d_{0.2}$ and $d_{0.35}$ to get quantities $x$ and $y$

$\left(\begin{array}{l}x \\ y\end{array}\right) \equiv\left(\begin{array}{cc}1 & 1.76 \\ -1 & 1.67\end{array}\right)\left(\begin{array}{c}d_{0.2} \\ d_{0.35}\end{array}\right)$,

which gives

$x=0.3836 \pm 0.0102$

$y=-0.0073 \pm 0.0070$.

The distance ratio $f \equiv D_{V}(0.35) / D_{V}(0.2)$ is given by

$f=\frac{1.67-1.76 y / x}{1+y / x} \simeq 1.67-8.94 y$,

where the last approximation neglects the small variations around the best-fit value of $x=0.3836$; these would come to 0.002 in $f$, which is well within the errors. Thus, $x$ is a measurement of distance for the concordance cosmology and $y$ is the deviation from the concordance distance ratio: $x$ is measured to about $2.7 \%$. $y$ is consistent with zero to within about $1 \sigma$.

To high accuracy, the constraint $x$ can be written as a constraint on the distance to some redshift $0.2<z<0.35$. In fact, $r_{s}\left(z_{d}\right) / D_{V}(0.275)$ predicts $x=d_{0.2}+1.76 d_{0.35}$ to a peak-topeak precision of $0.04 \%$ over the range $0.05<\Omega_{m}<1$ (assuming a flat cosmology with $w=-1$ ). Thus, we can quote the $x$ measurement as a measurement of $d_{0.275}$ and quote the $y$ measurement as a statistically independent measure of $f$.

For the best-fit solution we have $d_{0.275}=0.362 x$, giving

$d_{0.275}=0.1390 \pm 0.0037(2.7 \%)$.

We also have the statistically independent constraint

$f \equiv D_{V}(0.35) / D_{V}(0.2)=1.736 \pm 0.065$.

$f=1.67$ for our $\Lambda$ CDM concordance cosmology, while SCDM with $\Omega_{m}=1, \Omega_{\Lambda}=0$ has $f=1.55$, which is only $2.9 \sigma$ from this result. Our constraint from the distance ratio only separates the concordance model from $\Omega_{m}=1$ at $1.8 \sigma$, i.e., it is not a strong cosmological constraint, compared with the constraint on $d_{0.275}$.

\section{COSMOLOGICAL INTERPRETATION}

We now consider how our constraints can be mapped into the standard basis of cosmological parameters. From equation 6 of Eisenstein \& Hu (1998), the sound horizon can be approximated, around the WMAP5 best-fit location (Komatsu et al. 2009) as

$r_{s}\left(z_{d}\right)=153.5\left(\frac{\Omega_{b} h^{2}}{0.02273}\right)^{-0.134}\left(\frac{\Omega_{m} h^{2}}{0.1326}\right)^{-0.255}$ Mpc.

Setting $r_{s, f i d}=153.5 \mathrm{Mpc}$, and using Eq. (10) we have

$D_{V}(0.275)=(1104 \pm 30)\left[r_{s}\left(z_{d}\right) / r_{s, f i d}\left(z_{d}\right)\right] \mathrm{Mpc}$

$=(1104 \pm 30)\left(\frac{\Omega_{b} h^{2}}{0.02273}\right)^{-0.134}\left(\frac{\Omega_{m} h^{2}}{0.1326}\right)^{-0.255} \mathrm{Mpc},(13)$

(c) 0000 RAS, MNRAS 000, 000-000 
and $f=1.736 \pm 0.065$ as our two statistically independent constraints.

The constraint on $D_{V}(0.275)$, combined with a measurement of $\Omega_{m} h^{2}$ from WMAP5 (Hinshaw et al. 2009; Dunkley et al. 2009; Komatsu et al. 2009), is enough to measure $\Omega_{m}$ and $H_{0}$ given information about the distance scale from $z=0$ to $z=0.275$. If the distance measure were at $z=0$, then we would have a standard ruler defined by the CMB with which we could measure $H_{0}$, and combining this with $\Omega_{m} h^{2}$ would yield $\Omega_{m}$. In practice, one has to include the small corrections to $D_{V}(0.275)$ that arise from the lowredshift cosmology. Noting that $D_{V}(0.275)=757.4 h^{-1} \mathrm{Mpc}$ for a flat $\Omega_{m}=0.282 \Lambda \mathrm{CDM}$ cosmology, we can write $h=$ $\sqrt{\Omega_{m} h^{2}} / \sqrt{\Omega_{m}}$, and solve

$$
\begin{aligned}
\Omega_{m}=(0.282 & \pm 0.015)\left(\frac{\Omega_{m} h^{2}}{0.1326}\right)^{0.49} \\
& \times\left(\frac{D_{V}\left(z=0.275, \Omega_{m}=0.282\right)}{D_{V}(z=0.275)}\right)^{2},
\end{aligned}
$$

where we have dropped the dependence of the sound horizon on $\Omega_{b} h^{2}$, which the WMAP5 data already constrains to $0.5 \%, 5$ times below our statistical error.

We can perturb the ratio of distances around the best-fit $\Omega_{m}=$ 0.282 , to give

$$
\begin{aligned}
& \frac{D_{V}(z=0.275)}{D_{V}\left(z=0.275, \Omega_{m}=0.282\right)} \\
& \quad=\left(\frac{\Omega_{m}}{0.282}\right)^{-0.077}\left[1-0.108 \Omega_{k}-0.099(1+w)\right] .
\end{aligned}
$$

Using this approximation, we can manipulate Eq. (14) to give constraints on either $\Omega_{m}$ or $h$

$$
\begin{aligned}
\Omega_{m}=(0.282 & \pm 0.018)\left(\frac{\Omega_{m} h^{2}}{0.1326}\right)^{0.58} \\
& \times\left[1+0.25 \Omega_{k}+0.23(1+w)\right], \\
h=(0.686 & \mp 0.022)\left(\frac{\Omega_{m} h^{2}}{0.1326}\right)^{0.21} \\
& \times\left[1-0.13 \Omega_{k}-0.12(1+w)\right] .
\end{aligned}
$$

The additional uncertainty in $\Omega_{m}, \pm 0.018$ in Eq. 16 compared with \pm 0.15 in Eq. (14), is produced by the dependence of the distance ratio on $\Omega_{m}$. In Eqns. (16) \& (17), the uncertainty in the first terms are correlated so as to leave $\Omega_{m} h^{2}$ constant. One should additionally include the errors from $\Omega_{m} h^{2}, \Omega_{k}$, and $w$, although these are consistent between the two results.

Looking at the fractional error in $\Omega_{m}$, the contribution from the uncertainty in the SDSS acoustic scale is about $6 \%$, that from the uncertainty in $\Omega_{m} h^{2}$ is about $2 \%$, that from $w$ is about $3 \%$ if the error on $w$ is $10 \%$, and that from curvature is below $1 \%$ unless the cosmology is rather non-standard. Hence our result is still limited by the SDSS-II BAO data volume and not by our knowledge of the other cosmological parameters in Eq. (16). Of course, these expressions only hold for mild perturbations from the concordance cosmology; for other cases, one should return to the raw distance constraints. We note that these expressions have not used the angular acoustic scale in the CMB, so they are independent of what is happening with dark energy at $z>0.35$.

Fig. 5 shows the BAO constraints from Eq. 13 on $\Omega_{m}$ and $\Omega_{\Lambda}$ for $\Lambda \mathrm{CDM}$ cosmologies (upper panel), and on $\Omega_{m}$ and $w$ for flat models where constant $w \neq-1$ is allowed (lower panel). We take a Gaussian prior of $\Omega_{m} h^{2}=0.1326 \pm 0.0063$ and as-
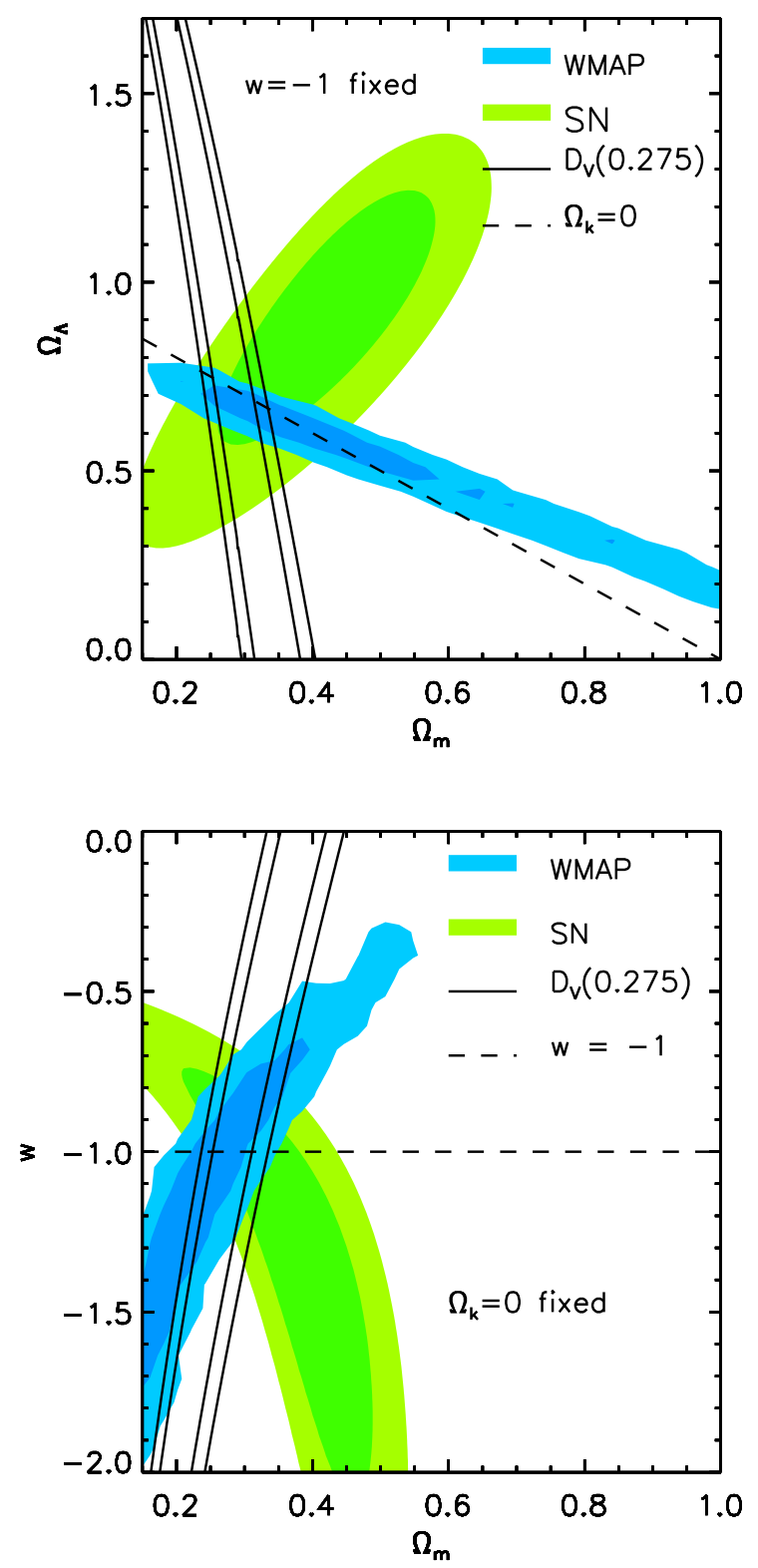

Figure 5. Cosmological constraints on $\Lambda \mathrm{CDM}$ cosmologies (upper panel) and flat CDM models where we allow $w$ to vary (lower panel), from WMAP5 (blue), Union supernova (green) and our constraint on $r_{s} / D_{V}(0.275)$ (solid contours). Contours are plotted for $-2 \ln \mathcal{L} / \mathcal{L}_{\text {true }}<2.3,6.0$, corresponding to $68 \%$ and $95 \%$ confidence intervals. The dashed lines show flat models (upper panel) and $\Lambda$ models (lower panel).

sume that the error on $\Omega_{b} h^{2}$ is negligible as the WMAP5 data already constrain it to $0.5 \%$ (Komatsu et al. 2009). These constraints exclude the angular acoustic scale in the $\mathrm{CMB}$, so they are independent of the dark energy behaviour at the redshifts beyond our sample. For comparison we plot the full WMAP5 constraints (Komatsu et al. 2009), which include the constraints on the distance to last scattering, and constraints from the Union supernova sample (Kowalski et al. 2008), which constrain angular diameter distance ratios up to $z \sim 1$. Results from full likelihood fits combining these data are presented in Section 9 


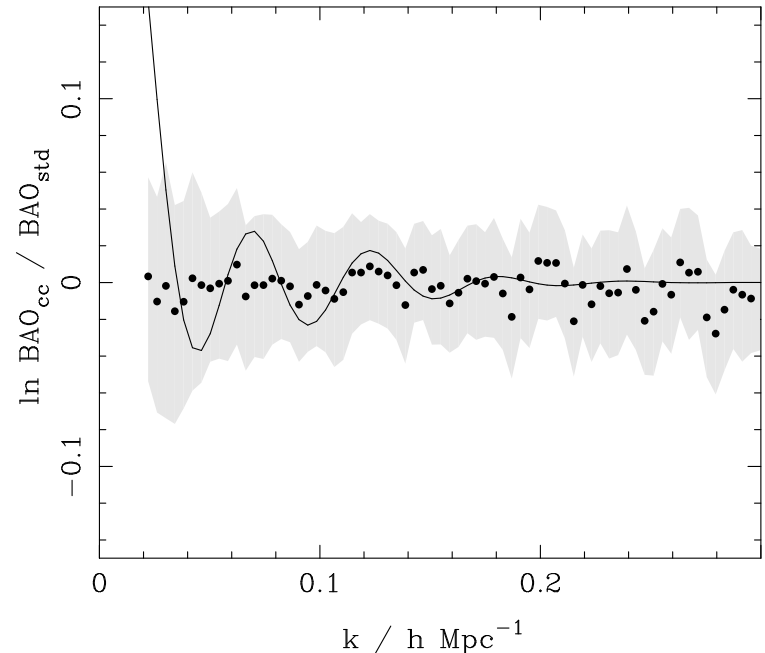

Figure 6. The log ratio between the BAO recovered from the SDSS LRG power spectrum and the power spectrum of the halo catalogue derived from the LRG sample as described by Reid et al. (2009) (solid circles). For comparison we plot the BAO expected for a flat $\Lambda$ CDM model with $\Omega_{m}=0.25, h=0.72, \& \Omega_{b} h^{2}=0.0223$ (solid line), and the errors on each measurement (grey shaded region). There are no oscillatory features induced by the cluster-collapse procedure, and the scatter is well within the errors.

\section{TESTING THE ROBUSTNESS OF THE RESULTS}

\subsection{The effect of redshift-space distortions}

We have fitted our spline $\times$ BAO model to the observed SDSS LRG power spectrum, as calculated by Reid et al. (2009), where the galaxy power spectrum and derived cosmological constraints are presented. Using numerical simulations, a scheme is presented in Reid et al. (2009) to recover the halo power spectrum from the LRG distribution by only keeping a single LRG within each halo. We have fitted both the galaxy and the halo power spectra with our spline $\times$ BAO model. The log ratio between the BAO recovered in the resulting fits is shown in Fig. 6 This shows that the clustercollapse correction for these galaxies results in a smooth change in the power spectrum on the scales fitted, and does not alter the position or amplitude of the BAO in a significant way.

Because of the different galaxy properties within the SDSS main galaxy sample, and the 2dFGRS, we do not attempt to correct for the more complicated distribution of galaxies within the haloes of that sample, and recover the halo power spectrum. In contrast, the halo occupation distribution of the SDSS LRGs is simple, in that there is only a single population of galaxies that are predominantly central rather than satellite galaxies in their hosting haloes (Reid et al. 2009). But we have seen that for LRGs, the correction is smooth, and we expect this to be true for the galaxies at $z<0.2$ as well.

\subsection{Sample selection}

We have run our full analysis pipeline using three subsamples of galaxies. Results from fits to $D_{V}(z)$ with two nodes are shown in Fig. 7 for different catalogues, given $r_{s}\left(z_{d}\right)=154.7 \mathrm{Mpc}$. The best-fit constraints for these models on $d_{z}$ are given in Table 3 Our default analysis is included in panel (a) for comparison. Here, we analyse data from the SDSS and the 2dFGRS, including the early SDSS data, where we cut the sample at the extinction-corrected

\begin{tabular}{lll}
\hline & $d_{0.2}$ & $d_{0.35}$ \\
\hline (a) default & $0.1905 \pm 0.0061$ & $0.1097 \pm 0.0036$ \\
(b) no early SDSS, 2dFGRS & $0.1923 \pm 0.0072$ & $0.1102 \pm 0.0041$ \\
(c) no 2dFGRS & $0.1907 \pm 0.0062$ & $0.1090 \pm 0.0036$ \\
(d) no early SDSS & $0.1917 \pm 0.0069$ & $0.1109 \pm 0.0044$ \\
(e) fit to three $P(k)$ & $0.1901 \pm 0.0066$ & $0.1080 \pm 0.0043$ \\
(f) original calibration & $0.1919 \pm 0.0071$ & $0.1094 \pm 0.0046$ \\
(g) varying $D_{\text {damp }}$ & $0.1918 \pm 0.0080$ & $0.1100 \pm 0.0048$ \\
(h) $\langle n(z)\rangle$ sampling galaxies & $0.1890 \pm 0.0068$ & $0.1102 \pm 0.0045$ \\
\hline
\end{tabular}

Table 3. Measurements of $d_{z} \equiv r_{s}\left(z_{d}\right) / D_{V}(z)$ at $z=0.2$ and $z=0.35$ from the different analysis runs described in the captions to Figs. $7 \& 8$

magnitude limit $r<17.5$. We compare with results obtained (b) excluding the early SDSS data and the 2dFGRS, (c) using just the SDSS data, and (d) excluding the early SDSS data but including the 2dFGRS. Including the early SDSS galaxies decreases the errors at redshift $z=0.2$ and $z=0.35$ by approximately $14 \%$. Including the 2dFGRS galaxies has a smaller effect, decreasing the error at $z=0.2$ by approximately $4 \%$. The parameters of the best-fit solutions do not move significantly with any of the sample changes: $d_{0.2}$ moves by a maximum of $0.3 \sigma$, while $d_{0.35}$ moves by a maximum of $0.2 \sigma$. The inclusion of the 2dFGRS actually moves the best-fit solution for $D_{V}(0.35) / D_{V}(0.2)$ slightly towards that of a concordance $\Lambda \mathrm{CDM}$ model.

\subsection{The number of redshift slices included}

We now consider the robustness of our fit to the number of redshift slices analysed. This test was performed on the conservative data sample, excluding the early SDSS data and the 2dFGRS. In our default analysis we fit power spectra calculated for six redshift slices, and the resulting likelihood surface for the late SDSS sample is shown in panel (b) of Fig. 7 For comparison, panel (e) of Fig. 8 shows the likelihood surface calculated using power spectra from only three redshift slices (details of the slices chosen are presented in Section 3). Because we are only fitting two $D_{V}(z)$ nodes, these should be constrained by our reduced fit using three redshift slices. Panel (e) of Fig. 8 shows that this is true, but comparison with panel (b) of Fig. 7 shows that the constraints are tighter if we model power spectra from six redshift slices. Clearly, extra information is available from the extra redshift slices, and we therefore fit to six redshift slices for our default analysis.

It is interesting to test if there is sufficient information to constrain the shape of $D_{V}(z)$ beyond our simple spline model with 2 nodes. Results from fits allowing four $D_{V}(z)$ nodes are shown in Fig. 9 There is a clear maximum in the slices through the likelihood surface close to the $\Lambda \mathrm{CDM}$ model, but the surface is noisy, and there are secondary maxima present. There is a strong degeneracy between $D_{V}(0.3)$ and $D_{V}(0.45)$, and between $D_{V}(0.1)$ and $D_{V}(0.2)$ : the data contain limited information to distinguish the shape of the distance-redshift relation between these redshifts. Consequently, we do not try to extract this information, instead concentrating on fits where there are only two nodes in $D_{V}(z)$.

\subsection{The covariance matrix}

Because we are analysing overlapping shells in redshift, the power spectra will be strongly correlated and the estimation of the covariance matrix will be in error if we do not have sufficient mock 

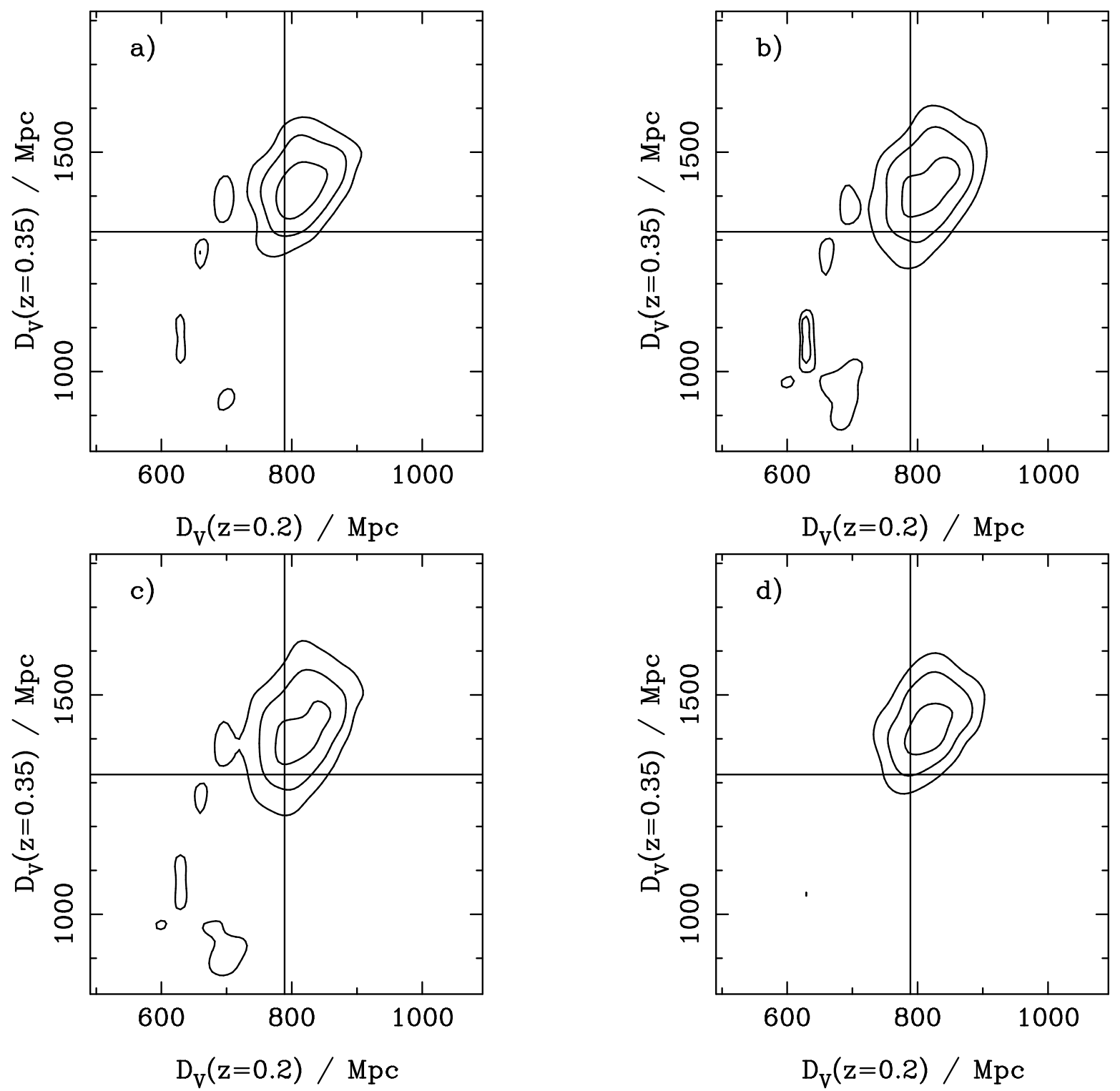

Figure 7. As Fig. 4 but now considering results from 4 choices of catalogue: (a) all SDSS and non-overlapping 2 dFGRS data, (b) excluding both the early SDSS data and 2dFGRS, (c) excluding the 2dFGRS, (d) excluding the early SDSS data.

catalogues. In order to test this, we have recalculated our covariance matrix using $1 / 3$ as many $\mathrm{LN}$ catalogues, and have used this matrix to recalculate the required corrections to the confidence intervals using independent sets of LN catalogues. We find consistent results in the factors required to match the confidence intervals to those expected for a multi-variate Gaussian distribution. We have also performed a full analysis using this reduced covariance matrix, and find results consistent with using our default covariance matrix.

\subsection{Calibration}

The likelihood surface shown in panel (f) of Fig. 8 was calculated using a SDSS galaxy sample with luminosities calibrated using the photometric calibration (Tucker et al. 2006), prior to the ubercalibration analysis (Padmanabhan et al 2008). This affects the calculation of the redshift completeness for any region observed, and also the luminosity-dependent weights applied to the SDSS galax- ies. The effect of this calibration change on our results is small, and there is no significant change between the likelihood surface in panel (f) of Fig. 8 and that in panel (b) of Fig. 7 where the ubercalibration data set was used.

\subsection{BAO damping scale}

Panel (g) of Fig. 8 shows the likelihood surface if we allow the BAO damping scale to be a free parameter in the fit, placing a simple Gaussian prior on its value $D_{\text {damp }}=10 \pm 5 h^{-1} \mathrm{Mpc}$. This prior on the BAO damping scale is conservative. From simulations, Reid et al. (2008) found $D_{\text {damp }}=9.2 \pm 1 h^{-1} \mathrm{Mpc}$, with no variation with redshift for $0<z<0.5$ for halo density fields, and $D_{\text {damp }}=9.7 \pm 1 h^{-1} \mathrm{Mpc}$ for density fields matched to the LRGs. The mild cosmological dependence suggested by Eisenstein et al. (2007) shows that the main cosmological dependence is through the linear growth rate; current constraints on $\sigma_{8}$ are much better 

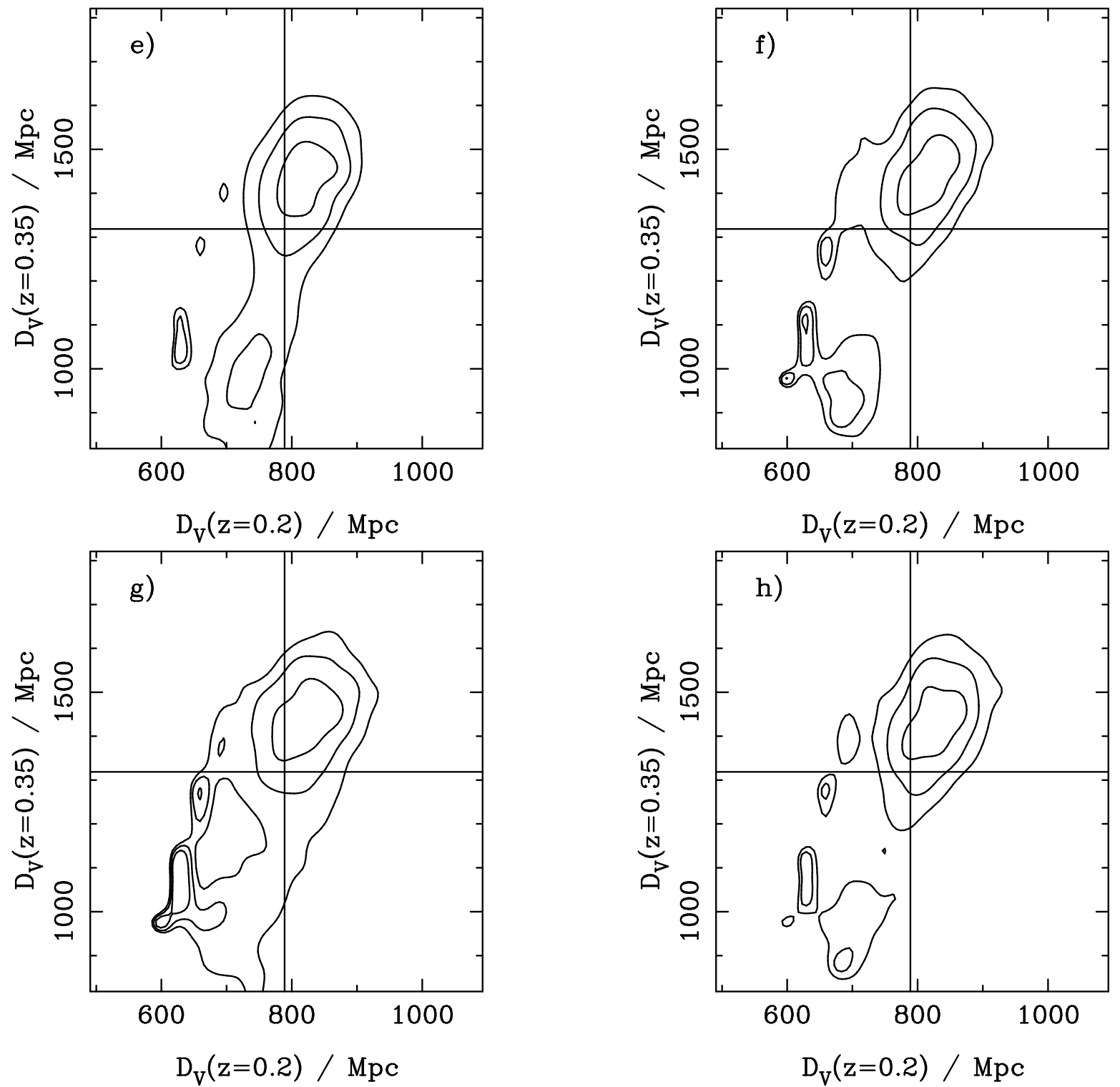

Figure 8. Likelihood contour plots as in Fig. 4 for the SDSS data, excluding the early data and the 2dFGRS, but now calculated for (e) fit to three power spectra, (f) old rather than uber-calibration. (g) allowing the BAO damping scale to vary with a simple Gaussian prior $D_{\text {damp }}=10 \pm 5 h^{-1} \mathrm{Mpc}$, (h) randomized galaxy redshifts used to give the expected radial galaxy distribution.

than that required to significantly change $D_{\text {damp }}$, and we consider $\pm 5 h^{-1} \mathrm{Mpc}$ to be a conservative prior. Allowing the damping scale to vary degrades the constraint, increasing the size of the parameter confidence regions. The best-fit solution does not move significantly, suggesting that our default assumption of a fixed damping scale is sufficiently accurate to current data precision.

\subsection{Radial galaxy distribution model}

Finally, analysis run (h) shows the constraints if we use a random catalogue where we randomly choose a galaxy redshift for each angular position chosen. i.e. to model the expected redshift distribution $\langle n(z)\rangle$, we sample from the galaxy redshift distribution. This test was designed to investigate the dependence of the analysis on how well we model the radial galaxy distribution. Randomly sampling galaxies to obtain this distribution, perfectly matches the red- shift distribution of the galaxies and that of the random catalogue used to define the survey region. In fact, we see no change in our results if we do this rather than using a smooth fit to the redshift distribution. This gives us confidence that our results are not sensitive to this modelling.

\subsection{Secondary likelihood maxima}

In the likelihood surfaces in Figs. 7 \& 8, we see secondary likelihood maxima, which appear to lie on a degeneracy stretching from $D_{V}(0.2)=700 \mathrm{Mpc}, D_{V}(0.35)=1500 \mathrm{Mpc}$ to $D_{V}(0.2)=$ $600 \mathrm{Mpc}, D_{V}(0.35)=1000 \mathrm{Mpc}$. These minor peaks in the likelihood, which appear as isolated islands in the likelihood surface are of lower significance than the strong peak close to the parameters of a concordance $\Lambda \mathrm{CDM}$ model. Tests have shown that the secondary peaks result from the interplay of two competing effects, which are 


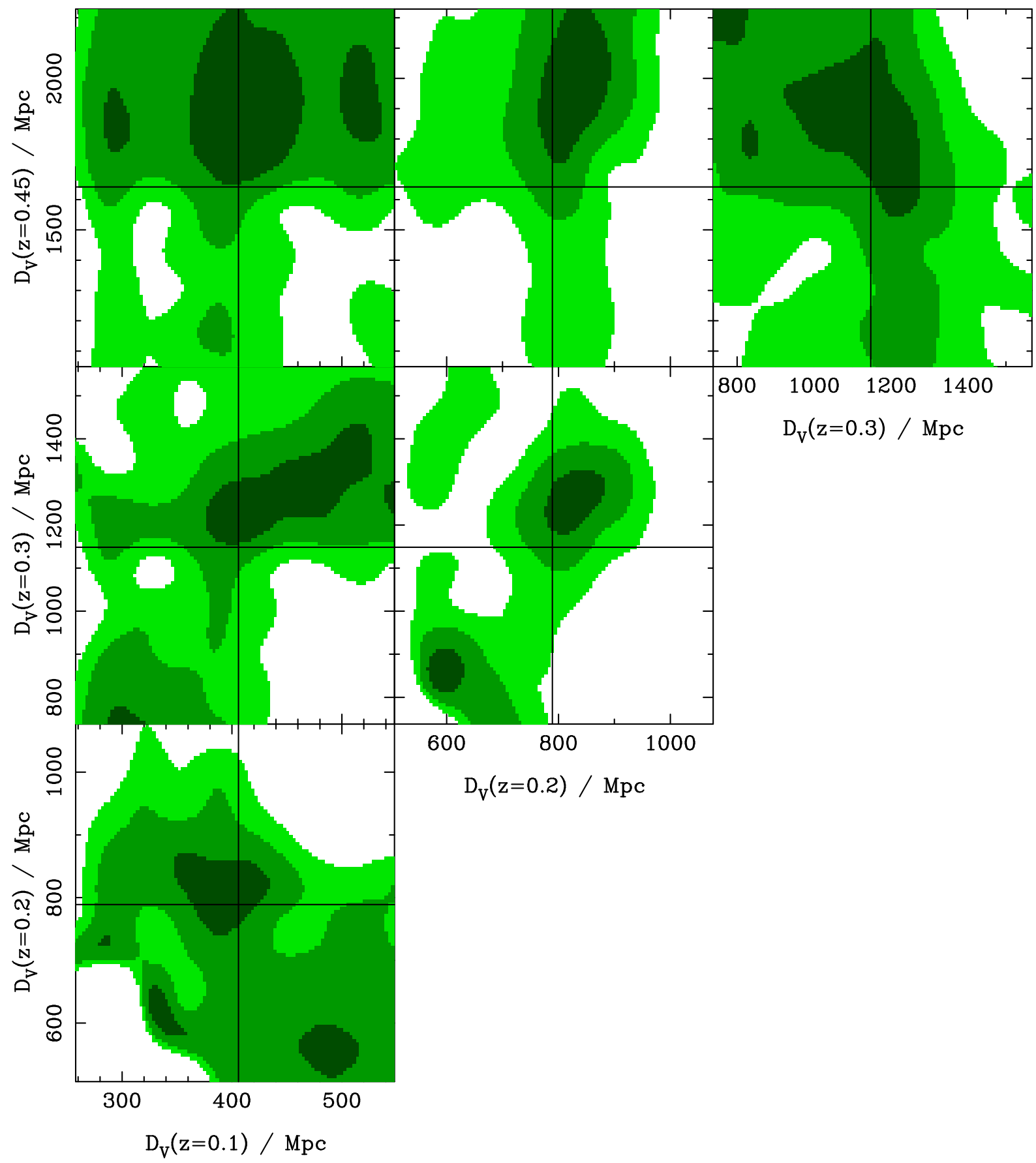

Figure 9. Contour plots showing slices through the likelihood for four $D_{V}(z)$ cubic spline nodes at $z=0.1, z=0.2 z=0.3$ and $z=0.45$, calculated for our default analysis using six power spectra, uber-calibration, and a fixed BAO damping scale of $D_{\text {damp }}=10 h^{-1}$ Mpc. Shaded regions are plotted for $-2 \ln \mathcal{L} / \mathcal{L}_{\text {true }}<2.3,6.0,9.3$, which for a multi-variate Gaussian distribution with two degrees of freedom correspond to $68 \%, 95 \%$ and $99 \%$ confidence intervals. Likelihoods were adjusted to match these Gaussian confidence intervals as described in Section 5 In each panel, the nodes that are not shown were fixed at the default $\Lambda \mathrm{CDM}\left(\Omega_{m}=0.25, \Omega_{\Lambda}=0.75\right)$ values. We use shaded regions in this plot to show the likelihood surface, compared with the contours in Figs. 7\& 8 because the likelihood surface is more complicated with four nodes, and the shading helps to distinguish peaks from troughs.

themselves a result of using the wrong cosmology to analyse the BAO. These are:

(i) A shift in the BAO position,

(ii) An increase in the width of the window associated with each band-power, caused by BAO in different redshift shells being outof-phase. This can smooth out the BAO signal.
Secondary maxima are produced where the BAO shift and the smoothing "balance". If we redo the analysis ignoring the second effect by assuming that the window function is a $\delta$-function centred on the peak, these secondary maxima are removed. 


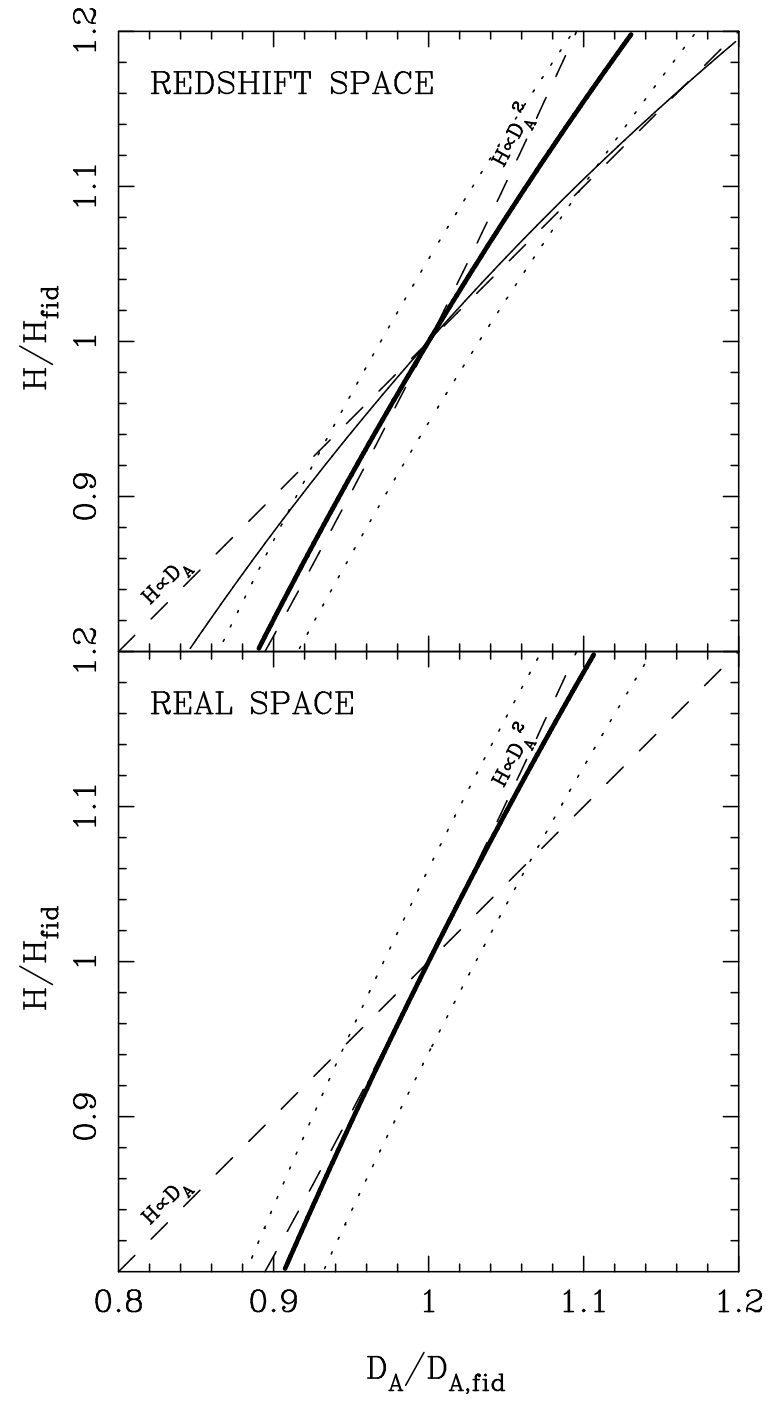

Figure 10. The expected shift recovered from an analysis of the BAO position in a spherically averaged galaxy power spectrum, if there are radial and angular distortions induced by assuming an incorrect cosmology when analysing the data. The thick solid contour shows no residual shift, while the dotted contours show a $1 \%$ shift. For comparison we plot the expected behaviour for an isotropic power spectrum $H(z) \propto D_{A}^{2}$, and for an increased importance of the radial distortion $H(z) \propto D_{A}$ (dashed lines). The top panel approximates redshift-space, by weighting the power in the spherical average by $\left(1+\beta \mu^{2}\right)^{2}$, with $\beta=0.25$, matching that expected for the SDSS LRGs, while the bottom panel does not include this weighting. For comparison, the thin solid contour in the top panel marks no residual shift for data with $\beta=1$, showing that we should expect the radial signal to increase in importance for such a sample.

\subsection{Dependency on $D_{V}$}

A possible concern about our method of analysis is that we assume a fiducial $\Lambda \mathrm{CDM}$ model to convert redshifts to comoving coordinate distances and measure the position of the $\mathrm{BAO}$ in the spherically-averaged power spectrum. If the true cosmological model has different angular diameter distance-redshift relation $D_{A}(z)$ and Hubble parameter $H(z)$ than this fiducial model, this would cause angular and radial distortions in the density field from which we estimate the power spectrum. By presenting results in terms of $D_{V}$ we remove the anisotropic information, and as- sume that the expected BAO position for all cosmological models is solely dependent on their predicted value of $D_{V}$. This must break down for models that behave very differently from our fiducial $\Lambda$ CDM model.

We now test the sensitivity of the assumption that the BAO position in the spherically averaged power spectrum only depends on $D_{V}$ for cosmological models that predict significant anisotropic distortions in the density field away from our fiducial model. To do this, we compute the shifts of the BAO position expected when one measures the spherically-averaged power in either real or redshift space for such models. To simplify the analysis, we assume that the BAO in the spherically averaged $P(k)$ will be shifted by the average of the shifts in $\mathbf{k}$ predicted over all angles: ie. our BAO fit recovers the weighted mean shift in the $3 \mathrm{D}$ power. In redshift-space we also follow the distant observer approximation, and assume that the angular dependence of the true 3D power spectrum is given by $\left(1+\beta \mu^{2}\right)^{2}$, where $\mu$ is the cosine of the angle to the line-of-sight and $\beta=\Omega_{m}^{0.55} / b$. The anisotropy in the observed power spectrum caused by redshift-space distortions will act as a weight when we spherically average.

For the SDSS LRGs, which provide most of our cosmological signal, we take an effective redshift of $z=0.35$, and assume a $\Lambda$ CDM model with $\Omega_{m}(z=0)=0.25$, giving $\Omega_{m}(z=$ $0.35)=0.45$. The LRGs are strongly biased and the model of Tegmark et al. (2004) gives an effective relative bias for our sample, which we correct for in the power spectrum calculation, of $\left\langle b / b_{*}\right\rangle=1.9$. Matching the normalisation of the measured LRG power spectrum (Reid et al. 2009) gives that $b_{*}=1.34$ assuming the LRG clustering is constant in comoving coordinates (e.g. Percival et al. 2007b), and that $\sigma_{8}$ (matter, $\left.z=0\right)=0.8$, so $\sigma_{8}($ matter, $z=0.35)=0.68$ Komatsu et al. 2009). This suggests that we should expect $\beta \sim 0.25$ for the LRG power spectrum, and we show contours calculated assuming $\beta=0.25$ in Fig. 10. which we compare with the prediction for $\beta=1$. Note that our luminosity-dependent weighting means that we are upweighting highly biased galaxies, and that our analysis will therefore have a smaller effective $\beta$ than analyses without such weighting, such as the measurements presented by Cabre \& Gaztanaga (2009).

Fig. 10 shows the relation between radial and angular distortions, $H / H_{\text {fid }}$ and $D_{A} / D_{A \text {,fid }}$, which give rise to zero and $\pm 1 \%$ shift in the spherical averaged power spectrum. Here, $H_{\text {fid }}$ is the fiducial value of $H$, and similarly for $D_{A}$. For general cosmological models, $H / H_{\text {fid }}$ and $D_{A} / D_{A \text {,fid }}$ will depend on redshift, so that the final effective shift will be an average over a trajectory in this diagram which is determined by the model to be tested. Fig. 10 also shows the expected line of zero average shift we would expect if the BAO position only depends on $D_{V}(z)$, which would lead to behaviour such that $H(z) \propto D_{A}^{2}$. For comparison, we show the prediction for a model with increased importance of the radial distortions, with $H(z) \propto D_{A}$. This is included because we would expect that the redshift-space distortions will increase the importance of the radial information. However, the $H(z) \propto D_{A}^{2}$ line is a significantly better fit, even in redshift-space. The $H(z) \propto D_{A}^{2}$ line does not cross the contours marking a $1 \%$ average shift for our redshift-space power spectrum, showing that the assumption that the recovered BAO position only depends on $D_{V}$ at most produces a $1 \%$ systematic in the best-fit for models with an anisotropy distortion away from our fiducial model of up to $20 \%$ in the radial direction. Such a $1 \%$ systematic shift, which requires a model that is extremely discrepant from $\Lambda \mathrm{CDM}$, is significantly below the statistical precision of our $2.7 \%$ accuracy distance measurement. It is 
therefore a reasonable approximation to use our measurements of $D_{V}$ to constrain a wide variety of cosmological models.

\section{COSMOLOGICAL PARAMETER CONSTRAINTS}

We now apply our full constraints to a cosmological parameter analysis. We assume that the likelihood of a model is given by a multi-variate Gaussian distribution around the $D_{V}(z)$ measurements given by Eq. (3), with the inverse covariance matrix of Eq. (5). Throughout this section we consider four models: a flat universe with a cosmological constant $(\Lambda \mathrm{CDM})$, a $\Lambda \mathrm{CDM}$ universe with curvature (o $\Lambda \mathrm{CDM})$, a flat universe with a dark energy component with constant equation of state $w$ (wCDM), and a wCDM universe with curvature (owCDM). This is the same model set considered by Reid et al. (2009). We use a modified version of COSMOMC (Lewis \& Bridle 2002) to perform the likelihood calculations.

\section{1 $\mathrm{SN}+\mathrm{BAO}+\mathrm{CMB}$ prior likelihood fits}

We first consider the constraints excluding the angular acoustic scale in the $\mathrm{CMB}$, in order to consider data that are independent of the dark energy behaviour at the redshifts beyond our sample. This is important because it ensures that our results only depend on the acceleration of the Universe at late times and so do not depend on so-called early dark energy models (Wetterich 1988; Ratra \& Peebles 1988; Zlatev et al. 1999; Steinhardt et al. 1999), which have non-negligible dark energy at early times. We take Gaussian priors $\Omega_{c} h^{2}=0.1099 \pm 0.0063$ and $\Omega_{b} h^{2}=0.02273 \pm$ 0.00061 from the CMB; these constraints from the ratio of peak heights in the WMAP5 data alone do not relax when $\Omega_{k}$ and $w$ are allowed to vary. We also impose weak priors on $-0.3<\Omega_{k}<0.3$ and $-3<w<0$. The parameter constraints from the combination of Union supernova (SN) (Kowalski et al. 2008) and BAO likelihoods with these priors are presented in Table 4 . The bestfit value of $\Omega_{m}$ ranges from 0.286 to 0.290 , with the $68 \%$ confidence interval, \pm 0.018 , while the mean value of $H_{0}$ varies between $67.8 \mathrm{~km} \mathrm{~s}^{-1} \mathrm{Mpc}^{-1}$ and $68.6 \mathrm{~km} \mathrm{~s}^{-1} \mathrm{Mpc}^{-1}$, and the $68 \%$ confidence interval remains $\pm 2.2 \mathrm{~km} \mathrm{~s}^{-1} \mathrm{Mpc}^{-1}$ throughout the four models. In Section 7 we derived BAO only constraints of \pm 0.018 on $\Omega_{m}$ and $\pm 2.2 \mathrm{~km} \mathrm{~s}^{-1} \mathrm{Mpc}^{-1}$ on $H_{0}$, for fixed $\Omega_{m} h^{2}$. If we include the $4.8 \%$ error on $\Omega_{m} h^{2}$ from the WMAP5 measurement, then we should expect these errors to increase to \pm 0.019 on $\Omega_{m}$ and $\pm 2.3 \mathrm{~km} \mathrm{~s}^{-1} \mathrm{Mpc}^{-1}$ on $H_{0}$. These agree perfectly with the COSMOMC results if we exclude the supernova data, so the small difference between the errors in Table 4 and those expected is caused by the supernova data helping to constrain $\Omega_{m}$ and $H_{0}$ slightly. Similarly, the best-fit values of these parameters agree for COSMOMC results excluding the supernova data. Comparison between Table 4 and Section 7 shows that the inclusion of the supernova data is moving the best-fit slightly: +0.004 in $\Omega_{m}$ and -0.5 in $H_{0}$ for the $\Lambda \mathrm{CDM}$ model. The COSMOMC analysis therefore validates the simple derivation presented in Section 7 In the space of models considered here, the BAO constraint on $D_{V}(0.275)$ already restricts $D_{V}(0.35) / D_{V}(0.2)$ to a much smaller region than our constraint in Eq. 111) allows. While the combination of these data and our priors are unable to constrain $\Omega_{k}, w$ is constrained at the \pm 0.11 level. For the owCDM model, the weak prior on $\Omega_{k}$ leads to an apparent constraint on $w$, but these errors depend strongly on the prior.

The data are compared with the best-fit $\Lambda \mathrm{CDM}$ model in Fig. 11 Three ways of considering the data constraints are shown

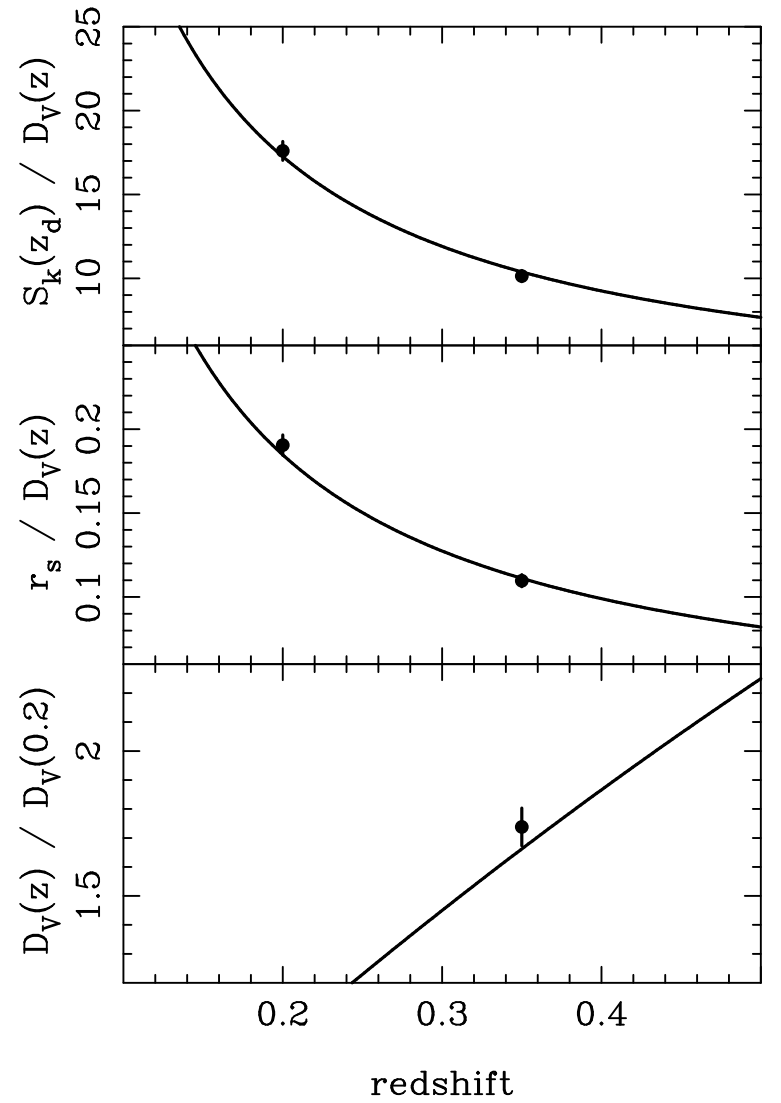

Figure 11. The BAO constraints (solid circles with $1 \sigma$ errors), compared with the best-fit $\Lambda$ CDM model. The three panels show different methods of using the data to constrain models.

in different panels. In the bottom panel we plot $D_{V}(z) / D_{V}(0.2)$, which corresponds to matching the geometry at $z=0.2$ and $z=0.35$ so the BAO match at these redshifts, without including information about the comoving position of the BAO. In the middle panel we plot $r_{s}\left(z_{d}\right) / D_{V}(z)$, where we now have to model the comoving sound horizon at the drag epoch. In the top panel we include a constraint on the sound horizon projected at the lastscattering surface as observed in the CMB. Marginalising over the set of flat $\Lambda$ CDM models constrained only by the WMAP5 data gives $r_{s}\left(z_{d}\right) / S_{k}\left(z_{d}\right)=0.010824 \pm 0.000023$, where $S_{k}\left(z_{d}\right)$ is the proper distance to the baryon-drag redshift $z_{d}=1020.5$, as measured by WMAP5 team (Komatsu et al. 2009). Ignoring the negligible error on this quantity, we combine with the BAO results to measure $S_{k}\left(z_{d}\right) / D_{V}(z)$. This effectively removes the dependence on the comoving sound horizon at the drag epoch, anchoring the BAO measurements at high redshift: here we have done this at the baryon-drag epoch so the CMB constraint has matched sound horizon and projection distance.

\subsection{CMB + BAO likelihood fits}

We now turn to the constraints from our BAO measurement combined with the full WMAP5 likelihood, including the constraint on $r_{s}\left(z_{d}\right) / D_{A}$ at the time of decoupling. While this extra constraint can break degeneracies between $\Omega_{m}, \Omega_{k}$, and $w$ inherent in our BAO constraints, the results are now sensitive to our assumption of a constant dark energy equation of state $w$ at $z>0.35$. Results for the four models are presented in Table 5 . 


\begin{tabular}{lllll}
\hline parameter & $\Lambda \mathrm{CDM}$ & $\mathrm{o} \Lambda \mathrm{CDM}$ & wCDM & owCDM \\
\hline$\Omega_{m}$ & $0.288 \pm 0.018$ & $0.286 \pm 0.018$ & $0.290_{-0.019}^{+0.018}$ & $0.286 \pm 0.018$ \\
$H_{0}$ & $68.1_{-2.1}^{+2.2}$ & $68.6 \pm 2.2$ & $67.8 \pm 2.2$ & $68.2 \pm 2.2$ \\
$\Omega_{k}$ & - & $-0.097 \pm 0.081$ & - & $-0.199_{-0.089}^{+0.080}$ \\
$w$ & - & - & $-0.97 \pm 0.11$ & $-0.838_{-0.084}^{+0.083}$ \\
$\Omega_{\Lambda}$ & $0.712 \pm 0.018$ & $0.811_{-0.085}^{+0.084}$ & $0.710_{-0.018}^{+0.019}$ & $0.913_{-0.082}^{+0.092}$ \\
\hline$d_{0.275}$ & $0.1381 \pm 0.0034$ & $0.1367 \pm 0.0036$ & $0.1384 \pm 0.0037$ & $0.1386 \pm 0.0037$ \\
$D_{V}(0.275)$ & $1111 \pm 31$ & $1120 \pm 33$ & $1109 \pm 32$ & $1108_{-33}^{+32}$ \\
$f$ & $1.662 \pm 0.004$ & $1.675 \pm 0.011$ & $1.659 \pm 0.011$ & $1.665 \pm 0.011$ \\
Age (Gyr) & $14.02_{-0.31}^{+0.32}$ & $14.43 \pm 0.48$ & $13.95 \pm 0.36$ & $14.38 \pm 0.44$ \\
\hline
\end{tabular}

Table 4. Marginalized one-dimensional constraints (68\%) for BAO+SN for flat $\Lambda \mathrm{CDM}, \Lambda \mathrm{CDM}$ with curvature (o $\Lambda \mathrm{CDM}$ ), flat $\mathrm{wCDM}$ (wCDM), and wCDM with curvature (owCDM). The non-standard cosmological parameters are $d_{0.275} \equiv r_{s}\left(z_{d}\right) / D_{V}(0.275)$ and $f \equiv D_{V}(0.35) / D_{V}(0.2)$. We have assumed priors of $\Omega_{c} h^{2}=0.1099 \pm 0.0063$ and $\Omega_{b} h^{2}=0.02273 \pm 0.00061$, consistent with WMAP5-only fits to all of the models considered here. We also impose weak flat priors of $-0.3<\Omega_{k}<0.3$ and $-3<w<0$.

\begin{tabular}{|c|c|c|c|c|c|c|c|}
\hline parameter & $\Lambda \mathrm{CDM}$ & $\mathrm{o} \Lambda \mathrm{CDM}$ & wCDM & owCDM & owCDM+SN & owCDM $+H_{0}$ & owCDM $+\mathrm{SN}+H_{0}$ \\
\hline$H_{0}$ & $70.1 \pm 1.5$ & $68.3_{-2.1}^{+2.2}$ & $69.3 \pm 3.9$ & $75.3 \pm 7.1$ & $67.6 \pm 2.2$ & $74.8 \pm 3.6$ & $69.5 \pm 2.0$ \\
\hline$w$ & - & - & $-0.97 \pm 0.17$ & $-1.53_{-0.50}^{+0.51}$ & $-0.97 \pm 0.10$ & $-1.49_{-0.31}^{+0.32}$ & $-1.00 \pm 0.10$ \\
\hline & $0.722 \pm 0.018$ & $0.724 \pm 0.019$ & $0.717 \pm 0.026$ & $0.772 \pm 0.048$ & $0.716 \pm 0.019$ & $0.773 \pm 0.029$ & $0.724 \pm 0.018$ \\
\hline $100 \Omega_{b} h^{2}$ & $2.267 \pm 0.058$ & $2.269 \pm 0.060$ & $2.275 \pm 0.061$ & $2.254_{-0.061}^{+0.062}$ & $2.271 \pm 0.061$ & $2.254_{-0.062}^{+0.061}$ & $2.284 \pm 0.061$ \\
\hline $\ln \left(10^{10} A_{05}\right)$ & $3.074_{-0.039}^{+0.040}$ & $3.060 \pm 0.042$ & $3.070 \pm 0.041$ & $3.062_{-0.043}^{+0.042}$ & $3.062_{-0.042}^{+0.041}$ & $3.062 \pm 0.042$ & $3.072 \pm 0.042$ \\
\hline$d_{0.275}$ & $0.1411 \pm 0.0030$ & $0.1387 \pm 0.0036$ & $0.1404_{-0.0035}^{+0.0036}$ & $0.1382 \pm 0.0037$ & $0.1379 \pm 0.0036$ & $\begin{array}{l}0.1387_{-0.0037}^{+0.0036} \\
\end{array}$ & $\begin{array}{l}0.1402_{-0.0034}^{+0.0033} \\
\end{array}$ \\
\hline$D_{V}(0.275)$ & $1080 \pm 18$ & $1110_{-31}^{+32}$ & $1089 \pm 31$ & $1111 \pm 33$ & $1115 \pm 32$ & $1107 \pm 31$ & $1091_{-28}^{+27}$ \\
\hline$f$ & $1.6645 \pm 0.0043$ & $1.6643 \pm 0.0045$ & $1.661 \pm 0.019$ & $1.72 \pm 0.056$ & $1.660 \pm 0.011$ & $1.7187_{-0.0334}^{+0.0337}$ & $1.6645 \pm 0.0107$ \\
\hline Age (Gyr) & $13.73 \pm 0.12$ & $14.08 \pm 0.33$ & $13.76_{-0.14}^{+0.15}$ & $14.49 \pm 0.52$ & $14.04 \pm 0.36$ & $14.48 \pm 0.48$ & $13.86_{-0.33}^{+0.34}$ \\
\hline
\end{tabular}

Table 5. Marginalized one-dimensional constraints (68\%) for WMAP5+BAO for flat $\Lambda \mathrm{CDM}, \Lambda \mathrm{CDM}$ with curvature (o $\Lambda \mathrm{CDM})$, flat wCDM (wCDM), wCDM with curvature (owCDM), and owCDM including constraints from supernovae. The non-standard cosmological parameters constrained by the BAO measurements are $d_{0.275} \equiv r_{s}\left(z_{d}\right) / D_{V}(0.275)$ and $f \equiv D_{V}(0.35) / D_{V}(0.2)$.

For the $\Lambda \mathrm{CDM}$ model, we find $\Omega_{m}=0.278 \pm 0.018$ and $H_{0}=70.1 \pm 1.5 \mathrm{~km} \mathrm{~s}^{-1} \mathrm{Mpc}^{-1}$, with errors significantly reduced compared to the WMAP5 alone analysis $\left(\Omega_{m}=0.258 \pm 0.03\right.$ and $\left.H_{0}=70.5_{-2.7}^{+2.6} \mathrm{~km} \mathrm{~s}^{-1} \mathrm{Mpc}^{-1}\right)$. Similar limits on $\Omega_{m}$ were obtained by Rozo et al. (2009) who used the maxBCG cluster abundance and weak-lensing mass measurements to similarly break the tight WMAP5 constraint on $\Omega_{m} h^{2}$.

Fig. 12] shows the impact of relaxing the flat, $\Lambda \mathrm{CDM}$ assumption. The WMAP5 results alone tightly constrain $\Omega_{m} h^{2}$ in all of these models (dashed lines), but low redshift information is necessary to constrain $\Omega_{m}$ and $H_{0}$ separately. Allowing $w \neq-1$ relaxes the constraint on $\Omega_{m}$ from the BAO measurement, and in addition allowing $\Omega_{k} \neq 0$ relaxes the constraint even further. The impact on the constraints on $\Omega_{m}$ and $H_{0}$ is shown in the lower right panel. All of the contours lie along the banana with $\Omega_{m} h^{2}$ fixed from the CMB.

In the $\mathrm{o} \Lambda \mathrm{CDM}$ model, the combination of scales measured by the $\mathrm{CMB}$ and the BAO tightly constrain the curvature of the universe: $\Omega_{k}=-0.007_{-0.007}^{+0.006}$. The constraints on $\Omega_{m}$ and $H_{0}$ in this model are well described by Eqns. (16) \& 17), while in the
wCDM cosmology they degrade because $w$ is not well-constrained by the low redshift BAO information alone.

When the parameter space is opened to both curvature and $w$, the WMAP5 data are not able to eliminate the degeneracy between $\Omega_{m}$ and $w$ in the BAO constraint. The constraints relax to $\Omega_{m}=0.240_{-0.043}^{+0.044}$ and $H_{0}=75.3 \pm 7.1 \mathrm{~km} \mathrm{~s}^{-1} \mathrm{Mpc}^{-1}$; $\Omega_{k}=-0.013 \pm 0.007$ is still well-constrained but $w$ is not (see Fig. 12). Including the constraints from the Union Supernova Sample breaks the remaining degeneracy, and we recover the tight constraints on $\Omega_{m}$ and $H_{0}$ given in Eqns. (16) \& (17). These constraints, and the relative degeneracies induced and broken by different data sets, are shown in Fig. 13 For each of the four models considered, the central values for $\Omega_{m}$ and $H_{0}$ change only slightly when the full WMAP5 likelihoods are used (Table 5 instead of priors on $\Omega_{b} h^{2}$ and $\Omega_{c} h^{2}$ in combination with the Union SN sample (Table 4).

Table 5 also lists the best fit cosmological age (i.e. time since the Big Bang) for different cosmologies and data sets. While the age is very well determined for $\Lambda \mathrm{CDM}$ and wCDM, there is a degeneracy between age and curvature that increases the uncertainties 

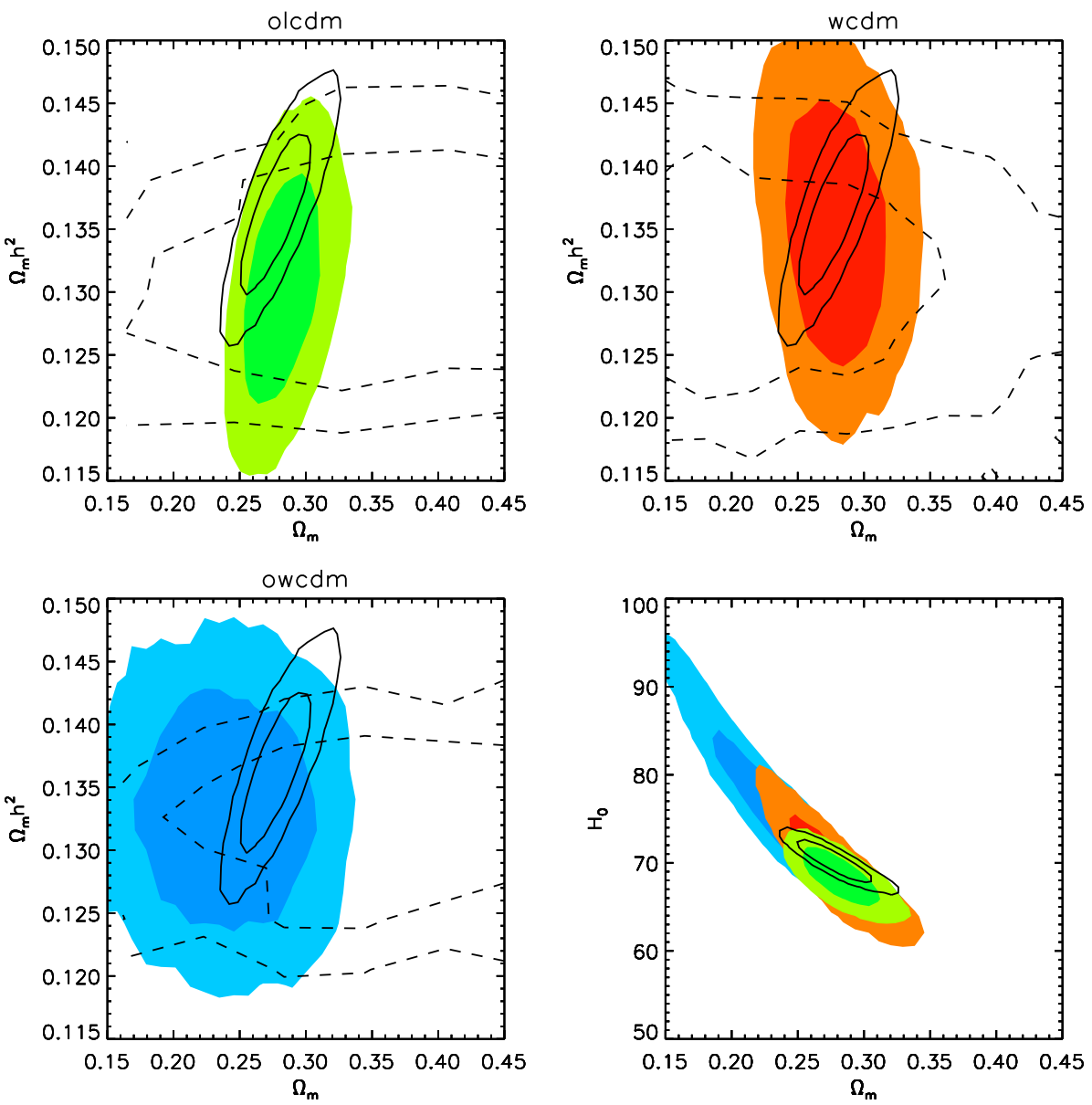

Figure 12. WMAP5+BAO constraints on $\Omega_{m} h^{2}, \Omega_{m}$, and $H_{0}$ for $\Lambda$ CDM (solid black contours), o $\Lambda$ CDM (shaded green contours), wCDM (shaded red contours), and owCDM (shaded blue contours) models. Throughout, the solid contours show WMAP5+LRG $\Lambda$ CDM constraints. The first three panels show WMAP5 only constraints (dashed contours) and WMAP5+BAO constraints (colored contours) in the $\Omega_{m} h^{2}-\Omega_{m}$ plane as the model is varied. In the lower right we show all constraints from WMAP5+BAO for all four models in the $\Omega_{m}-H_{0}$ plane, which lie within the tight $\Omega_{m} h^{2} \approx 0.133 \pm 0.006$ WMAP5-only constraints.

and allows for an older age in $\mathrm{o} \Lambda \mathrm{CDM}$ and owCDM information. Adding $\mathrm{SN}$ and $H_{0}$ measurements reduces these uncertainties and implies a best fit age of $13.86_{-0.33}^{+0.34} \mathrm{Gyr}$.

\subsection{Comparison with Riess et al. (2009) $H_{0}$}

Riess et al. (2009) recently released a new determination of the Hubble constant using a differential distance ladder: $H_{0}=74.2 \pm$ $3.6 \mathrm{~km} \mathrm{~s}^{-1} \mathrm{Mpc}^{-1}$. This value, as well as the values $H_{0} \approx$ $68 \mathrm{~km} \mathrm{~s}^{-1} \mathrm{Mpc}^{-1}$ determined in Table 4 using BAO, SN, and a WMAP5 prior on $\Omega_{c} h^{2}$ and $\Omega_{b} h^{2}$, are within $\sim 1 \sigma$ of the mean value determined from WMAP5+BAO in a $\Lambda$ CDM model, 70.1. In the wCDM model, combining this new $H_{0}$ with the WMAP5 likelihood constrains $w=-1.12 \pm 0.12$. In Table 5] we show MCMC results for the owCDM model for WMAP5+BAO $+H_{0}$ and WMAP5+BAO+H$+H_{0}+\mathrm{SN}$. In this model, the supernova data are more effective than $H_{0}$ at breaking the long degeneracy in the

1 We account for the small cosmology dependence in the $H_{0}$ constraint (seen as a slight degeneracy between $H_{0}$ and $w$ in fig. 14 of Riess et al. 2009) by considering it as a constraint on the inverse luminosity distance at the effective redshift $z=0.04$ (Riess private comm.)
WMAP5+BAO constraints. Combining WMAP5+BAO+SN+H , the mean parameters are quite close to $\Lambda$ CDM: $\Omega_{k}=-0.003 \pm$ 0.007 and $w=-1.00 \pm 0.10$, and $\Omega_{m}=0.279 \pm 0.016$ and $H_{0}=69.5 \pm 2.0 \mathrm{~km} \mathrm{~s}^{-1} \mathrm{Mpc}^{-1}$ are also well-constrained.

\section{COMPARISON WITH DR5 ANALYSES}

In Percival et al. (2007c), we presented BAO measurements calculated from fitting power spectra calculated for three samples drawn from the combined SDSS+2dFGRS catalogue, using the SDSS DR5 data. The full catalogue was split into galaxy populations, rather than redshift slices, corresponding to the SDSS LRGs, the 2dFGRS+SDSS main galaxies, and the combined sample. From this, we obtained the distance constraints $r_{s}\left(z_{d}\right) / D_{V}(0.2)=$ $0.1980 \pm 0.0058$ and $r_{s}\left(z_{d}\right) / D_{V}(0.35)=0.1094 \pm 0.0033$ with correlation coefficient 0.39 , which gives a distance ratio measurement of $D_{V}(0.35) / D_{V}(0.2)=1.812 \pm 0.062$. The concordance $\Lambda$ CDM value is $D_{V}(0.35) / D_{V}(0.2)=1.67$, measured using the SNLS supernova data, which is discrepant with the published DR5 $\mathrm{BAO}$ results at the $2.4 \sigma$ level. The analysis of mock catalogues presented in Section 5 showed that the cubic spline $\times$ BAO method underestimates the true distribution of recovered distances, given 

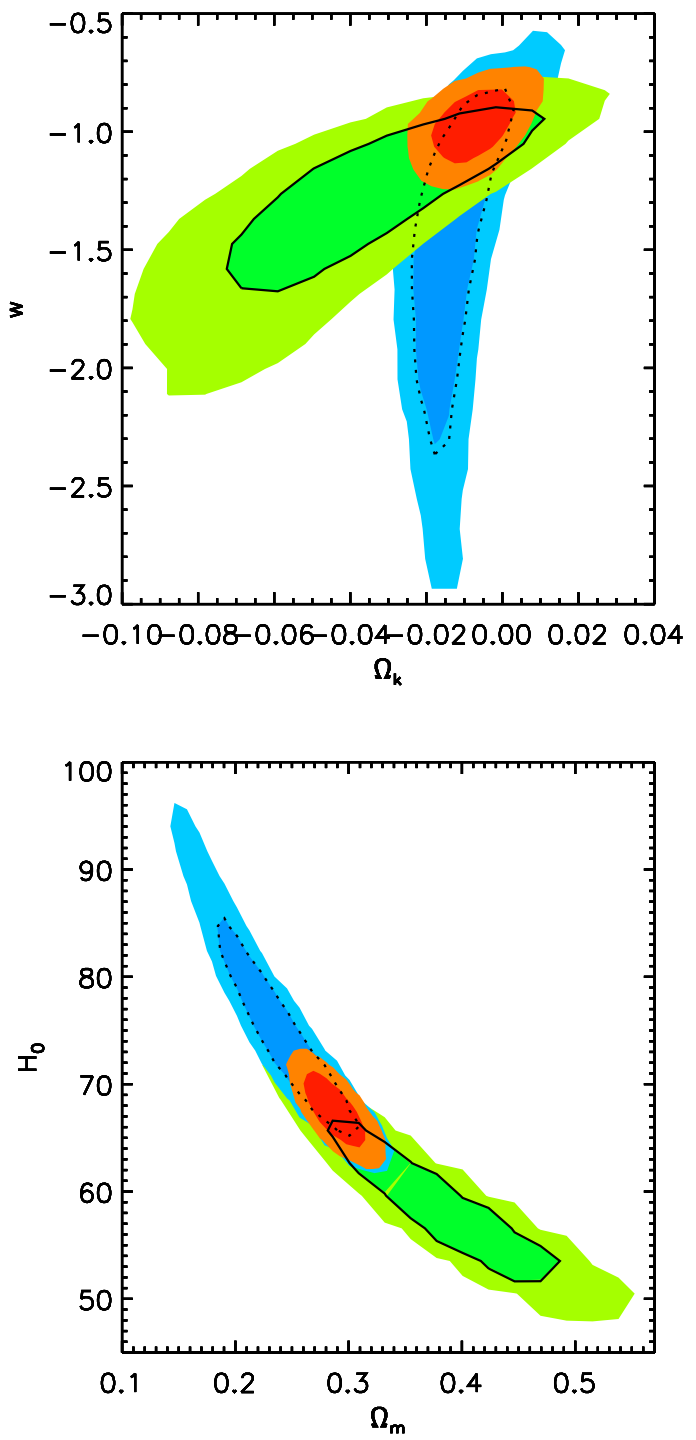

Figure 13. For the owCDM model we compare the constraints from WMAP5+BAO (blue contours), WMAP5+SN (green contours), and WMAP $+\mathrm{BAO}+\mathrm{SN}$ (red contours). Dashed and solid contours highlight the $68 \%$ confidence intervals for the WMAP5+BAO and WMAP5+SN models respectively.

noisy data, which produce a non-Gaussian likelihood surface. We should therefore increase the errors on the DR5 measurements of Percival et al. (2007c) by at least a factor of 1.14, which is the correction derived from the fits to three DR7 power spectra. If we do this, the revised DR5 constraints are $r_{s}\left(z_{d}\right) / D_{V}(0.2)=$ $0.1981 \pm 0.0071$ and $r_{s}\left(z_{d}\right) / D_{V}(0.35)=0.1094 \pm 0.0040$ with correlation coefficient 0.38 , which gives a distance ratio measurement of $D_{V}(0.35) / D_{V}(0.2)=1.813 \pm 0.073$. The discrepancy between the old DR5 constraints and the SNLS $\Lambda$ CDM value is reduced to $\lesssim 2 \sigma$. Because the DR5 data were noisier than the DR7 data, we should expect the likelihood surface to be less like a Gaussian prediction, and the correction actually should be slightly larger than that for the DR7 data.

Of all the changes implemented between this DR7 analysis and the analysis of the DR5 data, it was the increase in the number of random points used to quantify the survey geometry that had the most effect when comparing different catalogues. We now find consistent results, given in Table 3, for all catalogues and analysis variations presented in Section 8 . When translated into constraints on the distance ratio, for the full catalogue we find $D_{V}(0.35) / D_{V}(0.2)=1.736 \pm 0.065$. Using only 3 redshift slices we find $D_{V}(0.35) / D_{V}(0.2)=1.765 \pm 0.079$. If the $0.5 \sigma$ difference is not due to chance, the difference between these measurements could be caused by residual non-Gaussian scatter in the band powers. A scenario in which this is reduced by including fits to more redshift bins would then explain the observed trend. Excluding the 2dFGRS and early SDSS data, the constraint is reduced to $D_{V}(0.35) / D_{V}(0.2)=1.747 \pm 0.070$, which is consistent with the tighter constraint using all of the data.

Sanchez et al. (2009), who analysed the SDSS DR6 sample, speculated that the discrepancy could be caused by the Percival et al. (2007c) analysis fixing the BAO damping scale. However, in our current analysis, if we allow the BAO damping scale $D_{\text {damp }}$ to vary, the derived constraints on $D_{V}(0.35) / D_{V}(0.2)$ does not change significantly from that recovered in our default analysis. The mild discrepancy with $\Lambda$ CDM does not appear to be caused by fixing the damping scale. The change from photometric calibration to uber-calibration has a relatively minor effect on the distance ratio, which increases to $D_{V}(0.35) / D_{V}(0.2)=1.748 \pm 0.074$. Fig. 6 shows that the effect on the BAO of redshift-space distortions caused by the thermal motion of galaxies in clusters is similarly small. Linear redshiftspace distortions propagate the apparent position of galaxies along their velocity vector in a way that simply makes the field look more evolved than it is; they do not alter the positions of the BAO.

In conclusion, the significance of the discrepancy with flat $\Lambda \mathrm{CDM}$ models is reduced because of

(i) analysis of the non-Gaussian nature of the likelihood surface,

(ii) analysis of more redshift slices,

(iii) more accurate determination of the galaxy redshift distribution.

\section{DISCUSSION}

In this paper we have measured and analysed BAO from the SDSS DR7 sample, which represents the final data set observed using the original SDSS spectroscopic target selection algorithm. We have further developed the analysis method used by Percival et al. (2007c) to analyse the DR5 sample, including a faster method for the calculation of the window function (see Appendix $\mathrm{A}$, linking the cosmological model to be tested with the power spectrum bandpowers measured. This has enabled us to analyse power spectra calculated for six rather than three redshift slices, which would not have been possible using the old method.

In Section 6 we have shown how the distance-redshift constraints at $z=0.2$ and $z=0.35$ can be decomposed into a single distance constraint at $z=0.275$, and a "gradient" around this pivot given by $D_{V}(0.35) / D_{V}(0.2)$. This allows us to easily test the consistency of the $\Lambda$ CDM model without having to compare with additional data. For the best-fit flat $\Lambda$ CDM model that matches our constraint $d_{0.275}=0.1390 \pm 0.0037$, we find that our distanceratio measurement of $D_{V}(0.35) / D_{V}(0.2)=1.736 \pm 0.065$ is consistent at the $1.1 \sigma$ level.

Now that the SDSS-II sample is complete, the importance of including the $2 \mathrm{dFGRS}$ data is reduced, and the inclusion only decreases the low redshift $z=0.2$ distance error by $4 \%$. As we showed in Section 8.2 the inclusion of the 2dFGRS galaxies does 
not lead to the discrepancy with the $\Lambda$ CDM model: including the $2 \mathrm{dFGRS}$ brings our constraint slightly more into line with the predictions of $\Lambda \mathrm{CDM}$ models.

Of the cosmological parameter constraints presented in Tables 4 \& 5 , perhaps the most impressive are the constraints on $\Omega_{m}$ and $H_{0}$. For $\Lambda$ CDM models, fitting to BAO and Supernovae with priors on $\Omega_{m} h^{2}$ and $\Omega_{b} h^{2}$ gives $H_{0}$ to $3.2 \%$ and $\Omega_{m}$ to $6.4 \%$. These constraints are robust to the behaviour of the Universe at high redshift, as they are based only on the distance-redshift relation at redshift $z<0.35$ : we can allow $\Omega_{k} \neq 0$ and $w \neq-1$ with minimal effect. This weak dependence on $w$ and $\Omega_{k}$ was shown in Eqns. (16) \& (17) for the BAO data.

If we allow for the flatness constraint to be relaxed, then we obtain $\Omega_{k}=-0.007 \pm 0.007$ from the combination of BAO+WMAP5 data. A tight constraint was similarly obtained on $w=-0.97 \pm 0.17$ if we relax the $\Lambda$ constraint. If we allow both the curvature and the dark energy equation of state to vary, we must include more data to continue to break the degeneracy between the two parameters. We do so by including results from the Union SN dataset, giving us $\Omega_{k}=-0.006 \pm 0.008$ and $w=-0.97 \pm 0.10$, consistent with a flat $\Lambda \mathrm{CDM}$ model. If one allows only $w \neq-1 \mathrm{OR}$ $\Omega_{k} \neq 0$, then the combination of CMB, supernova and BAO data has an internal cross-check: opening two degrees of freedom from flat $\Lambda \mathrm{CDM}$ yields results that are consistent with flat $\Lambda \mathrm{CDM}$. We have also shown that our constraints are consistent with the recent re-determination of $H_{0}$ by Riess et al. (2009), and that combining this constraint with WMAP5, BAO, and SN in a model where both curvature and $w$ vary yields mean parameter values very close to $\Lambda \mathrm{CDM}$.

In a companion paper (Reid et al.2009), we consider the LRG sample in more detail. The LRGs are distributed in haloes in a simple way and we are able to extract the halo power spectrum from the data. In addition to fitting the BAO in this power spectrum, we are able to extract limited information about the shape of the power, which gives complementary constraints. A detailed comparison between the results from our fit to the BAO in redshift slices, performed in a cosmology model-independent way and including low-redshift galaxies, with the halo power spectrum of Reid et al. (2009) is presented in that paper, where excellent agreement is demonstrated. The data sets are correlated so they should not be used together to constrain cosmological models.

Our analysis highlights the importance of BAO as a key method for investigating cosmic acceleration, and shows that the method can already provide interesting cosmological constraints. Ongoing spectroscopic surveys aiming to use BAO to analyse dark energy include the Baryon Oscillation Spectroscopic Survay (BOSS; Schlegel et al. 2009a), the Hobby-Eberly Dark Energy Experiment (HETDEX; Hill et al. 2008) and the WiggleZ survey (Glazebrook et al. 2007). There are also plans for future surveys covering significantly larger volumes of the Universe, and therefore observing the BAO signal with higher precision such as the Square Kilometer Array (SKA: www.skatelescope.org), and the Joint Dark Energy Mission (JDEM: jdem.gsfc.nasa.gov) and European Space Agency Euclid satellite mission concepts, or the Big Baryon Oscillation Spectroscopic Survay (BigBOSS; Schlegel et al. 2009b). Photometric surveys such as the Dark Energy Survey (DES: www.darkenergysurvey.org), the Panoramic Survey Telescope \& Rapid Response System (PanStarrs: pan-starrs.ifa.hawaii.edu) and the Large Synoptic Survey Telescope (LSST: www. lsst.org) will find BAO using photometric redshifts. All of these surveys will measure BAO at higher redshifts than those analysed in our paper using SDSS-II data: if dark energy does not have a simple explanation, then comparison between future high redshift results and our current understanding of the low-redshift Universe from SDSS-II will provide an interesting test of these models.

\section{ACKNOWLEDGEMENTS}

WJP is grateful for support from the UK Science and Technology Facilities Council, the Leverhulme trust and the European Research Council. DJE was supported by National Science Foundation grant AST-0707225 and NASA grant NNX07AC51G. Simulated catalogues were calculated and analysed using the COSMOS Altix 3700 supercomputer, a UK-CCC facility supported by HEFCE and STFC in cooperation with CGI/Intel. WJP would like to thank Tamara M. Davis, Ravi K. Sheth, Roman Scoccimarro, Eyal Kazin, Taka Matsubara and the referee, Fergus Simpson, for useful interactions.

The 2dF Galaxy Redshift Survey was undertaken using the Two-degree Field facility on the $3.9 \mathrm{~m}$ Anglo-Australian Telescope. The success of the survey was made possible by the dedicated efforts of the staff of the Anglo-Australian Observatory, both in creating the $2 \mathrm{dF}$ instrument and in supporting the survey observations.

Funding for the SDSS and SDSS-II has been provided by the Alfred P. Sloan Foundation, the Participating Institutions, the National Science Foundation, the U.S. Department of Energy, the National Aeronautics and Space Administration, the Japanese Monbukagakusho, the Max Planck Society, and the Higher Education Funding Council for England. The SDSS Web Site is http://www.sdss.org/

The SDSS is managed by the Astrophysical Research Consortium for the Participating Institutions. The Participating Institutions are the American Museum of Natural History, Astrophysical Institute Potsdam, University of Basel, Cambridge University, Case Western Reserve University, University of Chicago, Drexel University, Fermilab, the Institute for Advanced Study, the Japan Participation Group, Johns Hopkins University, the Joint Institute for Nuclear Astrophysics, the Kavli Institute for Particle Astrophysics and Cosmology, the Korean Scientist Group, the Chinese Academy of Sciences (LAMOST), Los Alamos National Laboratory, the Max-Planck-Institute for Astronomy (MPIA), the MaxPlanck-Institute for Astrophysics (MPA), New Mexico State University, Ohio State University, University of Pittsburgh, University of Portsmouth, Princeton University, the United States Naval Observatory, and the University of Washington.

\section{REFERENCES}

Abazajian K., et al., 2009, ApJS, 182, 543

Adelman-McCarthy J., et al., 2006, ApJS, 162, 38

Adelman-McCarthy J., et al., 2007, ApJS, 172, 634

Adelman-McCarthy J., et al., 2008, ApJS, 175, 297

Albrecht A., et al., 2006, Report of the Dark Energy Task Force, [astro-ph/0609591]

Angulo R.E., Baugh C.M., Frenk C.S., Lacey C.G., 2008, MNRAS, 383, 755

Bharadwaj S., 1996, ApJ, 472, 1

Blake C. \& Glazebrook K., 2003, ApJ, 594, 665

Blake C., Collister A., Bridle S., Lahav O., 2007, MNRAS, 374, 1527 
Blanton M.R., Lin H., Lupton R.H., Maley F.M., Young N., Zehavi I., Loveday J., 2003a, AJ, 125, 2276

Blanton M.R., et al., 2003b, ApJ, 592, 819

Bond, J.R. \& Efstathiou, G. 1984, ApJ, 285, L45

Bond, J.R., \& Efstathiou, G., 1987, MNRAS, 226, 655

Cabre A., Gaztanaga E., 2009, MNRAS, 396, 1119

Cole S., et al., 2005, MNRAS, 362, 505

Coles P., Jones B., 1991, MNRAS, 248, 1

Colless M., et al., 2003, astro-ph/0306581

Cresswell J.G., Percival W.J., 2009, MNRAS, 392, 682

Crocce M., Scoccimarro R., 2006, PRD73, 063519

Crocce M., Scoccimarro R., 2008, PRD77, 023533

Dunkley, J., et al. 2009, ApJS, 180, 306

Eisenstein D.J., Hu W., 1998, ApJ, 496, 605

Eisenstein D.J., et al., 2001, AJ, 122, 2267

Eisenstein D.J., et al., 2005, ApJ, 633, 560

Eisenstein D.J., Seo H.-J., White M., 2007, ApJ, 664, 660

Feldman H.A., Kaiser N., Peacock J.A., 1994, MNRAS, 426, 23

Fukugita M., Ichikawa T., Gunn J.E., Doi M., Shimasaku K., Schneider D.P., 1996, AJ, 111, 1748

Gaztanaga E., Cabre A., Hui L., 2008, [|arXiv:0807.3551|]

Glazebrook K., et al., 2007, ASP conference series Vol 379, 72, [[astro-ph/0701876]]

Goldberg D.M., Strauss M.A., 1998, ApJ, 495, 29

Górski, K.M., Hivon E., Banday A.J., Wandelt B.D., Hansen F.K., Reinecke M., Bartelmann M., 2005, ApJ, 622, 759

Gunn J.E., et al., 1998, AJ, 116, 3040

Gunn J.E., et al., 2006, AJ, 131, 2332

Hill G.J., et al., 2008, ASP conference series Vol. 399, 115, [|arXiv:0806.0183|]

Hinshaw, G., et al. 2009, ApJS, 180, 225

Hogg D.W., Finkbeiner D.P., Schlegel D.J., Gunn J.E., 2001, AJ, 122,2129

Holtzman J.A. 1989, ApJS, 71,1

Hu W., Haiman Z., 2003, PRD, 68, 3004

Huetsi G., 2006, A\&A, 449, 891

Kim J., Park C., Gott R., Dubinski J., 2009, ApJ, 701, 1547

Kazin E., et al., 2009, ApJ submitted, arXiv:0908.2598

Komatsu, E., et al. 2009, ApJS, 180, 330

Kowalski, M., et al. 2008, ApJ, 686, 749

Ivezic Z., et al., 2004, AN, 325, 583

Lewis A., Challinor A., Lasenby A., 2000, ApJ, 538, 473

Lewis A., Bridle S., 2002, PRD66, 103511

Lupton R.H., Gunn J.E., Szalay A.S., 1999, AJ, 118, 1406

Lupton R., Gunn J.E., Ivezic Z., Knapp G.R., Kent S., Yasuda N., 2001, in ASP Conf. Ser. 238, Astronomical Data Analysis Software and Systems X, ed. F.R. Harnden Jr, F.A. Primini, H.E. Payne (San Francisco Astr. Spc. Pac.); astro-ph/0101420

Matsubara T., 2004, ApJ, 615, 573

Matsubara T., 2008a, PRD, 77, 063530

Matsubara T., 2008b, PRD, 78, 083519

Meiksin A., White M. \& Peacock J.A., 1999, MNRAS, 304, 851

Padmanabhan N., et al., 2007, MNRAS, 378, 852

Padmanabhan N., et al., 2008, ApJ, 674, 1217

Padmanabhan N., White M., Cohn J.D., PRD submitted, arXiv:0812.2905

Padmanabhan N., White M., et al., 2009, PRD, 80, 063508

Peebles, P. J. E. \& Yu J. T., 1970, ApJ, 162, 815

Percival W.J., et al., 2001, MNRAS, 327, 1297

Percival W.J., Verde L., Peacock J.A., 2004, MNRAS, 347, 645

Percival W.J., et al., 2007a, ApJ, 657, 51

Percival W.J., et al., 2007b, ApJ, 657, 645
Percival W.J., Cole S., Eisenstein D., Nichol R., Peacock J.A., Pope A., Szalay A., 2007c, MNRAS, 381, 1053

Pier J.R., Munn J.A., Hindsley R.B., Hennessy G.S., Kent S.M., Lupton R.H., Ivezi Z., 2003, AJ, 125, 1559

Press W.H., Teukolsky S,A., Vetterling W.T., Flannery B.P., 1992, Numerical recipes in C. The art of scientific computing, Second edition, Cambridge: University Press.

Ratra B., Peebles P.J.E., 1988, PRD, 37, 3406

Reid B., Spergel D.N., Bode P., 2008, ApJ, 702, 249

Reid B., et al., 2009, MNRAS submitted, [|arXiv:0907.1659|]

Riess, A.G., et al., 2009, ApJ, 699, 539

Rozo E., et al., 2009, [|arXiv:0902.3702|]

Sanchez A.G., Baugh C.M., Angulo R., 2008, MNRAS, 390, 1470

Sanchez A.G., Crocce M., Cabre A., Baugh C.M., Gaztanaga E., 2009, MNRAS submitted, [|arXiv:0901.2570]]

Seo H.-J., Eisenstein D.J., 2003, ApJ, 598, 720

Schlegel D., White M., Eisenstein D.J., 2009a, [|arXiv:0902.4680]]

Schlegel D., et al., 2009b, [|arXiv:0904.0468|]

Seo H.-J., Eisenstein D.J., 2005, ApJ, 633, 575

Seo H.-J., Eisenstein D.J., 2007, ApJ, 665, 14

Seo H.-J., Siegel E.R., Eisenstein D.J., White M., 2008, ApJ, 636, 16

Silk J., 1968, ApJ, 151, 459

Smith J.A., et al., 2002, AJ, 123, 2121

Smith R.E., Scoccimarro R., Sheth R.K., 2007, PRD, 75, 063512

Smith R.E., Scoccimarro R., Sheth R.K., 2008, PRD, 77, 043525

Springel V., et al., 2005, Nature, 435, 629

Steinhardt P.J., Wang L.-M., Zlatev I., 1999, PRD, 59, 123504

Stoughton C., et al., 2002, AJ, 123, 485

Strauss M.A., et al., 2002, AJ, 124, 1810

Sunyaev, R.A., \& Zel'dovich, Ya.B., 1970, Astrophys. \& Space Science, 7, 3

Swanson, M. E. C., Tegmark, M., Blanton, M., \& Zehavi, I. 2008, MNRAS, 385, 1635

Tegmark, M., et al., 2004, ApJ, 606, 702

Tegmark, M., et al., 2006, PRD, 74, 123507

Tucker D.L., et al., 2006, AN, 327, 821

Wetterich C., 1988, B302, 645

White M., 2005, Astroparticlephys., 24, 334

York D.G., et al., 2000, AJ, 120, 1579

Zlatev I., Wang L.-M., Steinhardt P.J., 1999, PRL, 82, 896

Zehavi I., et al., 2002, ApJ, 571, 172

Zehavi, I., et al., 2005, ApJ, 630, 1

\section{APPENDIX A: CALCULATION OF THE WINDOW FUNCTION}

In this Appendix, we describe the method used to calculate the mapping between the power spectra in the "true" cosmology to be tested, and the measured, or observed, power spectra where a $\Lambda$ CDM model was used to convert redshifts to distances. This window function includes both the effect of the survey geometry and the mapping between cosmological models. As described by Percival et al. (2007c), we should expect the observed power spectrum to be a convolution of the true power spectrum with a window function.

$P(k)_{\mathrm{obs}}=\int d k^{\prime} W\left(k, k^{\prime}\right) P\left(k^{\prime}\right)_{\text {true }}$

The goal of this section is to introduce a fast method by which $W\left(k, k^{\prime}\right)$ can be calculated for any model. 


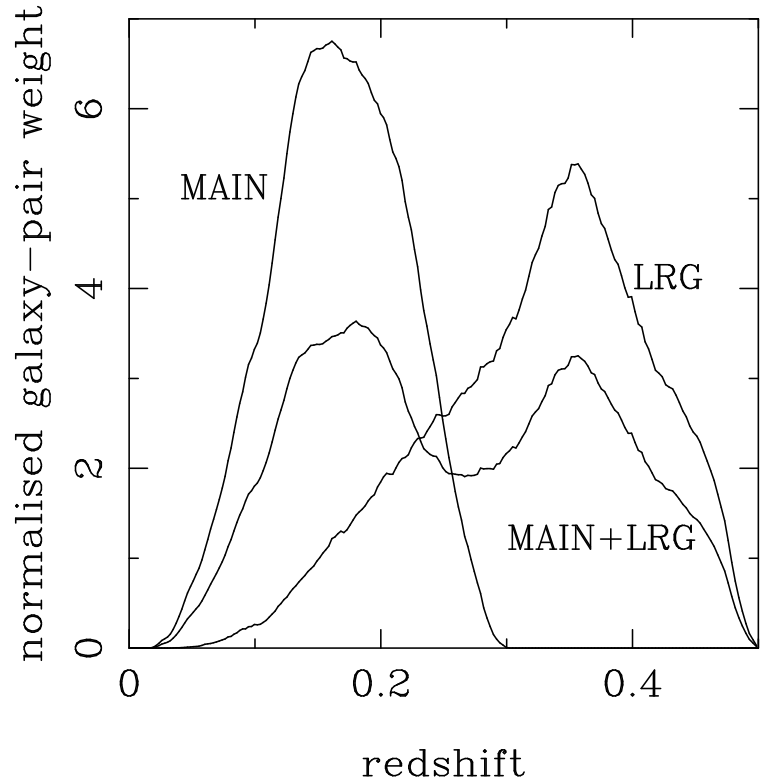

Figure A1. The redshift dependence of galaxy pair-weights for the SDSS DR7 LRG and main galaxy samples, and from the combination of the two. These curves were calculated assuming a flat $\Lambda \mathrm{CDM}$ cosmology with $\Omega_{m}=0.25, h=0.72, \& \Omega_{b} h^{2}=0.0223$.

In Percival et al. (2007c), this window function was calculated using Monte-Carlo realisations of Gaussian density fields, created assuming the cosmological model to be tested. These fields were then distorted as if they had been analysed assuming a $\Lambda \mathrm{CDM}$ model, and the power spectrum was calculated and compared with that input. Using a large number of simple input power spectra, we were able to construct the window function from this comparison. This procedure required significant computational resources as many density fields were needed in order to accurately measure the window function, limiting the number of models that could be tested. In particular, we were only able to consider cubic spline models of $D_{V}(z)$ with two nodes to three power spectra. With a faster window function calculation, we can include more nodes, and fit to more power spectra.

For a survey covering a thin shell, the window function relating true and observed power is an offset delta function

$W\left(k, k^{\prime}\right)=\delta_{D}\left[k / k^{\prime}-\epsilon\right]$,

where $\epsilon=d_{p}$ (true) $/ d_{p}$ (obs) is the ratio of proper distances in the true and observed cosmologies. Here we are simply stretching the true survey prior to measuring the power spectrum.

The obvious extension to surveys over a range of redshifts is to split the sample into $i$ redshift shells, and to approximate the window function as

$W\left(k, k^{\prime}\right)=\sum_{i} \delta_{D}\left[k / k^{\prime}-\epsilon_{i}\right] w_{i}$,

where $w_{i}$ is the weighted number of galaxy pairs in redshift shell $i$. Because we are now considering a broad survey, this pair weight is a function of pair separation. In this paper, we bin pairs of galaxies with comoving separation $90 h^{-1} \mathrm{Mpc}<d_{\Lambda \mathrm{CDM}}<$ $130 h^{-1} \mathrm{Mpc}$, where $d_{\Lambda \mathrm{CDM}}$ is the comoving distance in the $\Lambda \mathrm{CDM}$ cosmology used to convert galaxy redshifts to distances. The bin size was chosen to approximately match the BAO scale. For the SDSS LRG, main galaxy and combined samples, the galaxy pair-weights are shown in Fig. A1 We also need to allow for dif-

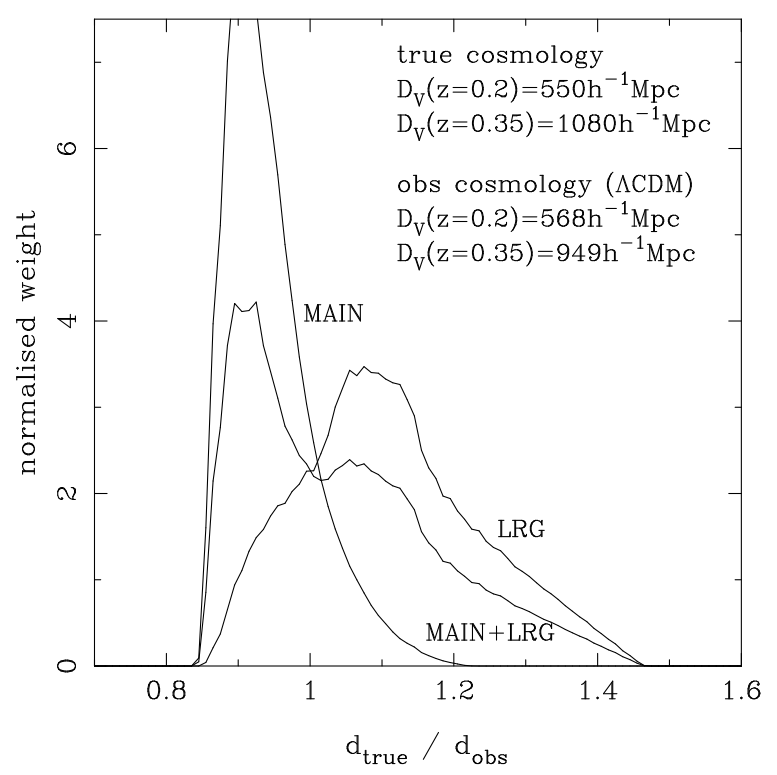

Figure A2. Galaxy pair-weights for the SDSS DR7 LRG and main galaxy samples, and from the combination of the two, as a function of comoving distance shifts. These were calculated assuming that a flat $\Lambda$ CDM cosmology with $\Omega_{m}=0.25, h=0.72, \& \Omega_{b} h^{2}=0.0223$ was used to analyse the data, while the BAO are present in a true cosmological model with distance-redshift relation defined by a cubic spline in $D_{V}(z)$ with nodes at $z=0.2$ and $z=0.35$, with amplitude as shown in the plot.

ferences in the orientation of galaxy pairs, as the distribution of $\epsilon_{i}$ should allow the galaxy pairs to be of all orientations. Including radial separations introduces an asymmetric convolution for $\epsilon_{i}$, and we have found that this needs to be included in order to provide approximately the correct window function shapes. Note that Eq. (A3) is exact when there is a perfect dilation of scale between the true and observed cosmologies: such stretching of the windows can be perfectly represented by this equation.

For each "true" cosmology to be tested, we can calculate the shift in scale that stretches each pair of galaxies because we do not measure BAO using this model. We have to allow for the angular shift caused by a change in $D_{A}(z)$ and the radial shift caused by the true and observed $H(z)$ being different. An example of the weighted distribution of "shifts" expected for a model cosmology defined by a cubic spline in $D_{V}(z)$ with two nodes at $z=0.2$ and $z=0.35$ is shown in Fig. A2 Here the true cosmology has a distance-redshift relation given by a spline fit to $D_{V}(z)$, with nodes $D_{V}(z=0.2)=550 h^{-1} \mathrm{Mpc}$, and $D_{V}(z=$ $0.35)=1080 h^{-1} \mathrm{Mpc}$. The $\Lambda \mathrm{CDM}$ values are $D_{V}(z=0.2)=$ $568 h^{-1} \mathrm{Mpc}$, and $D_{V}(z=0.35)=949 h^{-1} \mathrm{Mpc}$, so at redshift $z=0.2$, BAO in the true cosmology are stretched to larger scales by the analysis method, while those at redshift $z=0.35$ are compressed to smaller scales. For the SDSS main galaxies, with median redshift close to $z \simeq 0.2, d_{\text {true }} / d_{\text {obs }}<1$, while for the LRGs, with median redshift $z \simeq 0.35, d_{\text {true }} / d_{\text {obs }}>1$.

For each "true" cosmological model, the window function relating the true and observed power spectra was calculated by convolving the standard window function for the $\Lambda \mathrm{CDM}$ model, by the distribution of shifts such as that shown in Fig. A2 For the models shown in Fig. A2 we have calculated the window function using the approximate method outlined in this Appendix, and using the Monte-Carlo method described by Percival et al. (2007c). A comparison of the windows is presented in Fig. A3 Reasonable 

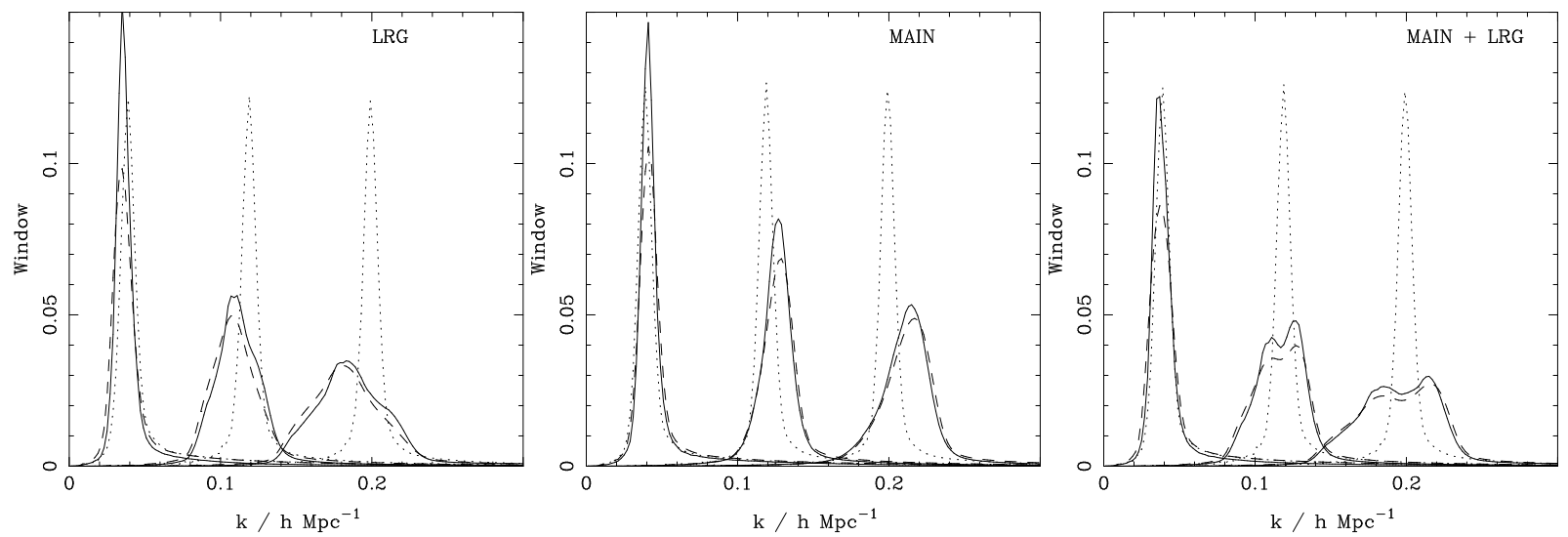

Figure A3. Window functions for three values of $k$, calculated for the SDSS LRG, main galaxy and combined catalogues. Dotted lines represent the windows for our fiducial $\Lambda \mathrm{CDM}$ cosmology. Solid and dashed lines show the window functions, if the true cosmology were different, but the data were analysed assuming that the fiducial $\Lambda \mathrm{CDM}$ cosmology is correct. The solid lines were calculated using the procedure outlined in this Appendix. Dashed lines were calculated using the Monte-Carlo procedure of Percival et al. 2007c).

agreement is found between the different methods: it is clear that the approximate method of splitting into shells recovers the main features of the window function. The agreement is not perfect, as expected given the approximate nature of our calculation. Because we analyse the data using a $\Lambda \mathrm{CDM}$ model, the window will be correct for this model, and will only deviate if we consider significantly different distance-redshift relations. 